ESAIM: COCV 21 (2015) 217-246

DOI: $10.1051 / \mathrm{cocv} / 2014026$
ESAIM: Control, Optimisation and Calculus of Variations

www.esaim-cocv.org

\title{
RESONANT EFFECTS IN RANDOM DIELECTRIC STRUCTURES
}

\author{
Guy Bouchitté ${ }^{1}$, Christophe Bourel $^{2}$ And Luigi Manca ${ }^{3}$
}

\begin{abstract}
In [G. Bouchitté and D. Felbacq, C. R. Math. Acad. Sci. Paris 339 (2004) 377-382; D. Felbacq and G. Bouchitté, Phys. Rev. Lett. 94 (2005) 183902; D. Felbacq and G. Bouchitté, New J. Phys. 7 (2005) 159], a theory for artificial magnetism in two-dimensional photonic crystals has been developed for large wavelength using homogenization techniques. In this paper we pursue this approach within a rigorous stochastic framework: dielectric parallel nanorods are randomly disposed, each of them having, up to a large scaling factor, a random permittivity $\varepsilon(\omega)$ whose law is represented by a density on a window $\Delta_{h}=\left[a^{-}, a^{+}\right] \times[0, h]$ of the complex plane. We give precise conditions on the initial probability law (permittivity, radius and position of the rods) under which the homogenization process can be performed leading to a deterministic dispersion law for the effective permeability with possibly negative real part. Subsequently a limit analysis $h \rightarrow 0$, accounting a density law of $\varepsilon$ which concentrates on the real axis, reveals singular behavior due to the presence of resonances in the microstructure.
\end{abstract}

Mathematics Subject Classification. 35B27, 35Q60, 35Q61, 35R60, 60H25, 78M35, 78M40.

Received September 10, 2013. Revised April 13, 2014

Published online December 9, 2014.

\section{INTRODUCTION}

Physical background. The paper is motivated by recents studies on metamaterials which are artificial structures that can be described, in certain ranges of frequencies, by homogeneous parameters. These materials enjoy very unusual properties not encountered in natural materials. In particular, it is possible to design devices in such a way that their effective permittivity and permeability be both negative, resulting in an effective negative index, as studied years ago by Veselago [25]. Several geometries have been proposed, notably the split ring resonators structure, that can produce artificial magnetism $[5,18,20]$. Such a structure can be combined with a metallic wire mesh structure in order to produce meanwhile a negative permittivity $[2,13,15,16,22-24]$. In reference [21], O'Brien and Pendry also suggested that it is possible to obtain an artificial magnetic activity in photonic crystals made of dielectric rods with a strong permittivity. In that case, there are internal resonances (Mie resonances) in the rods at wavelengths which are large compared with the period of the structure. Around the resonances, the magnetic field is strongly localized inside the rods: this produces a loop of displacement current inducing a microscopic magnetic moment. All the microscopic moments add up to a collective macroscopic

Keywords and phrases. Stochastic homogenization, photonic crystals, metamaterials, micro-resonators, effective tensors, dynamical system.

1 IMATH, Université du Sud Toulon-Var, 83957 La Garde cedex, France. bouchitte@univ-tln.fr

2 LMPA, Université du littoral côte d'Opale, 62228 Calais cedex, France. bourel@univ-littoral.fr

3 LAMA, Université de Marne la Vallée, 77454 Marne la Vallée cedex 2, France. luigi.manca@univ-mlv.fr 
moment, resulting in artificial magnetism. In $[11,14]$ a rigorous justification for this phenomenon was proposed in the particular case of a photonic crystal made of infinitely long parallel rods illuminated by a transverse magnetic polarized incident field: by using a two-scale analysis and by exploiting the spectral decomposition of the 2D-Dirichlet-Laplace operator on the section of the rods, it was possible to establish a frequency dependent effective permeability law with sign changing real part. Next in [14], the influence of random variations in the position and permittivity of the rods has been studied numerically. For the well posedness of the diffraction problem, a small dissipation (positive imaginary part) has been assumed for the permittivity of the random rods and, as expected, the influence of this dissipation combined with disorder tends to attenuate the resonance effects.

In this paper we will study the previous dielectric rod structure in a full rigorous random framework. The incident magnetic field is assumed to be polarized in the vertical direction $e_{3}$ and parallel to the rods. By invariance in the $x_{3}$-direction, the diffration problem can be therefore reduced in a $2 \mathrm{D}$ framework. The generalization of our results to a full 3D-framework is much more involved and could be foreseen as a challenging perspective (see [6] in the periodic deterministic case).

Description of the random obstacle and scaling. We consider a bounded domain $\mathcal{B} \subset \mathbb{R}^{2}$ in which small circular inclusions are randomly disposed. These inclusions occupy a subregion $\mathcal{D}_{\eta}(\omega) \subset \mathcal{B}$ representing the sections of the highly dielectric rods. Here $\eta$ is an infinitesimal quantity accounting the distance between inclusions while $\omega$ represents the random dependence in contrast with the standard notation for the angular frequency commonly used in waves theory.

More precisely, to every event $\omega$ (in a probability space $\Omega$ defined later), we associate a sequence of disks $\left\{B\left(\boldsymbol{\theta}_{j}(\omega), \boldsymbol{\rho}_{j}(\omega)\right) ; j \in \mathbb{Z}^{2}\right\}$ whose centers $\boldsymbol{\theta}_{j}(\omega)$ and radius $\boldsymbol{\rho}_{j}(\omega)$ are chosen randomly independent and so that $B\left(\boldsymbol{\theta}_{j}(\omega), \boldsymbol{\rho}_{j}(\omega)\right) \subset \subset Y:=(0,1)^{2}$. Then, letting $\boldsymbol{y}(\omega) \in Y$ be a uniformly distributed random translation of the unit lattice of $\mathbb{R}^{2}$, we define the following random subset of $\mathbb{R}^{2}$ :

$$
\mathcal{D}(\omega):=\bigcup_{j \in \mathbb{Z}^{2}} \mathcal{D}^{j}(\omega), \quad \mathcal{D}^{j}(\omega):=j-\boldsymbol{y}(\omega)+B\left(\boldsymbol{\theta}_{j}(\omega), \boldsymbol{\rho}_{j}(\omega)\right)
$$

Then after a $\eta$ dilatation, we determine a fine perforated domain in $\mathcal{B}$ by setting

$$
\begin{gathered}
\mathcal{D}_{\eta}(\omega):=\bigcup_{j \in J_{\eta}(\omega)} \mathcal{D}_{\eta}^{j}(\omega), \quad \mathcal{D}_{\eta}^{j}(\omega):=\eta \mathcal{D}^{j}(\omega) \\
\text { where } \quad J_{\eta}(\omega)=\left\{j \in \mathbb{Z}^{2} \mid \eta(j-\boldsymbol{y}(\omega)+Y) \subset \mathcal{B}\right\} .
\end{gathered}
$$

For technical reasons (see Rem. 2.1), we also assume that there exists $\delta \in\left(0, \frac{1}{2}\right)$ such that $\operatorname{dist}\left(\boldsymbol{\theta}_{j}(\omega), \partial Y\right)>$ $\rho_{j}+\delta$ for all $j \in \mathbb{Z}^{2}$ and all $\omega \in \Omega$. The scattering obstacle $\mathcal{D}_{\eta}(\omega)$ is depicted in Figure 1 .

A fundamental issue is now to choose a suitable scaling for the permittivity contrast between the rods and the surrounding matrix. In order that resonances occur for an incident wavelength which is large with respect to the radius of the rods (of order $\eta$ ), we need that the relative permittivity in them to be of order $\eta^{-2}$. We refer for instance to $[11,14,21]$ for further comments on this choice. To simplify we will assume that the permittivity in the matrix $\mathcal{B} \backslash \mathcal{D}_{\eta}(\omega)$ is the same as in the vacuum. In contrast in each rod, namely on $\mathcal{D}_{\eta}^{j}(\omega)$, we set the relative permittivity to be $\eta^{-2} \varepsilon_{j}(\omega)$ where $\left\{\varepsilon_{j}(\omega), j \in J_{\eta}(\omega)\right\}$ are mutually independent equi-distributed random variables ranging in the set $\mathbb{C}^{+}$of complex numbers with positive imaginary part. An important observation is that in our model the so called "optical radius" of the dielectric rods, given by the real numbers $\eta \boldsymbol{\rho}_{j}(\omega) \sqrt{\Re\left(\varepsilon_{j}(\omega) \eta^{-2}\right)}$, is randomly distributed but independent of $\eta$.

Diffraction problem. The set of random rods is illuminated by a monochromatic incident wave traveling in the $H \|$ mode. The incident magnetic field is therefore of the form $H^{\text {inc }}(x, t)=u^{\text {inc }}\left(x_{1}, x_{2}\right) \mathrm{e}^{-i \nu t} e_{3}$ where $\nu$ denotes the angular frequency and $u^{\text {inc }}$ solves Helmholtz equation $\Delta u^{\text {inc }}+k_{0}^{2} u^{\text {inc }}=0$ in $\mathbb{R}^{2}$ where $k_{0}:=\nu / c=2 \pi / \lambda$ is the wave number ( $c$ is the light speed in the vacuum and $\lambda$ the wavelength). 


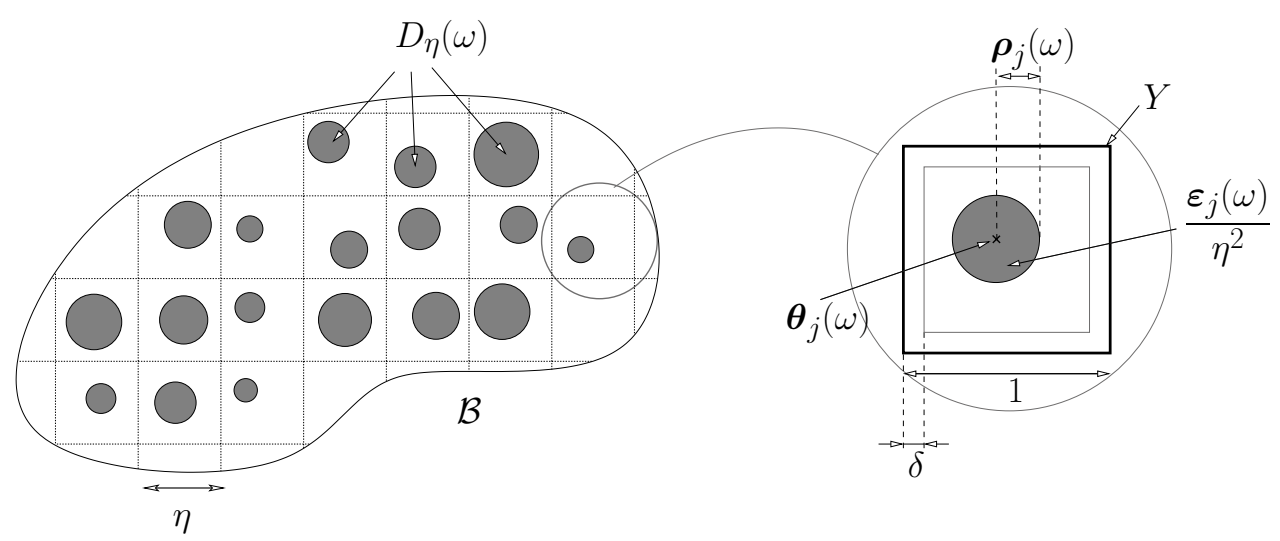

FiguRE 1. Description of the random obstacle.

Here and in what follows, in order to simplify the mathematical framework, the unit of length has been normalized so that roughly $\lambda$ represents the ratio between the physical wavelength and the size of the obstacle $\mathcal{B}$. Therefore our normalized $k_{0}$ and $\lambda$ are dimensionless quantities.

Then as usual the total electromagnetic field solving Maxwell's system is completely determined by the magnetic field $H_{\eta}(x, t)=u_{\eta}\left(x_{1}, x_{2}\right) \mathrm{e}^{-i \nu t} e_{3}$ where $u_{\eta}$ solves

$$
\operatorname{div}\left(a_{\eta}(x, \omega) \nabla u_{\eta}(x, \omega)\right)+k_{0}^{2} u_{\eta}(x, \omega)=0 \quad x \in \mathbb{R}^{2}, \omega \in \Omega .
$$

Here the complex function $a_{\eta}(x, \omega)$ represents the inverse of the local permittivity. In view of the scaling adopted before, it is given by

$$
a_{\eta}(x, \omega)=1_{\mathbb{R}^{2} \backslash \mathcal{D}_{\eta}(\omega)}(x)+\sum_{j \in J_{\eta}(\omega)} \frac{\eta^{2}}{\varepsilon_{j}(\omega)} 1_{\mathcal{D}_{\eta}^{j}(\omega)}(x) .
$$

In addition the diffracted field $u_{\eta}^{d}=u_{\eta}-u^{\text {inc }}$ satisfies the outgoing Sommerfeld radiation condition

$$
\lim _{r \rightarrow \infty} \sqrt{r}\left(\partial u_{\eta}^{d} / \partial r-i k_{0} u_{\eta}^{d}\right)=0 .
$$

Let us emphasize that the randomness of the system and all the physical informations of the model are determined by the probability law $\mathbf{p}$ on $Y \times[0,1 / 2] \times \mathbb{C}^{+}$shared by the random triples $\left(\boldsymbol{\theta}_{j}(\omega), \boldsymbol{\rho}_{j}(\omega), \boldsymbol{\varepsilon}_{j}(\omega)\right)$. We point out also that in this model no magnetic activity is present in the rods namely their permeability is taken to be the same as in the vacuum.

Asymptotic issues. We are interested in the behavior of diffraction problem (1.4) for infinitesimal values of parameter $\eta$. In particular we want to show that, under suitable conditions on the probability law $\mathbf{p}$, the solution $u_{\eta}(x, \omega)$ does converge almost surely to a deterministic limit. We expect this limit to be characterized as the unique solution of a new diffraction problem in which the obstacle $\mathcal{B}$ is filled with an homogeneous material characterized by suitable effective permeability and permittivity tensors.

Our goal is twofold:

- First we want to derive conditions on the given probability law $\mathbf{p}$ under which uniform $L^{2}$-estimates hold for the sequence $\left\{u_{\eta}\right\}$ allowing to undergo the homogenization process in a similar way as in the deterministic case studied in [3]. 
- Second we wish to study the homogenized dispersion law for the effective permittivity in term of $\mathbf{p}$. In particular an important issue is the influence of the disorder when the support of the probability law of the $\varepsilon_{j}(\omega)$ 's concentrates along the real axis. Surprisingly the limit anaysis will reveal that the homogenized permeability law retains a non vanishing dissipative part.

Outline. The paper is organized as follows. The main homogenization result is stated in Section 2 after introducing suitable notations and definitions. In Section 3, we analyze the effective permeability law emphasizing on the combined role of resonances and randomness. In particular, we discuss the behavior of the limit field with respect to some critical choice of the law $\mathbf{p}$. Then after a detailed review of the stochastic framework in Section 4, we present in Section 5 the two-scale analysis of the diffraction problem. Section 6 is devoted to the proof of the main result (Thm. 2.3) followed by a technical Appendix.

\section{The Main homogenization Result}

Notations. We denote by $\left\{e_{1}, e_{2}\right\}$ the canonical base of $\mathbb{R}^{2}$. Sometimes $e_{1}, e_{2}$ will be identified as elements of $\mathbb{Z}^{2}$. Given $x, y \in \mathbb{R}^{2},|x|$ denotes the Euclidean norm and $x \cdot y$ the scalar product. Accordingly, for every $r>0$, $B(x, r)$ is the open ball centered in $x$ of radius $r$ and we denote $B_{r}:=B(0, r)=\{|x|<r\}$. For every open set $A \subset \mathbb{R}^{2}, C_{c}^{\infty}(A)$ stands for the space of $C^{\infty}$ complex valued functions whose support is a compact subset of $A$. Given a complex number $z \in \mathbb{C}$, we denote by $\Re(z)$ and $\Im(z)$ its real and imaginary parts. We denote $\mathbb{C}^{+}:=\{z \in \mathbb{C}: \Im(z) \geq 0\}$. Given $\delta$ a fixed parameter such that $0<\delta<1 / 2$, we define

$$
M=\left\{(\theta, \rho, \varepsilon) \in Y \times[0,1 / 2] \times \mathbb{C}^{+}: \operatorname{dist}(\theta, \partial Y) \geq \rho+\delta\right\} .
$$

Eventually we will consider $Y:=(0,1)^{2}$ as the unit periodicity cell in $\mathbb{R}^{2}$. Accordingly we denote by $W_{\sharp}^{1,2}(Y)$ the set of restrictions to $Y$ of functions in $W_{\text {loc }}^{1,2}\left(\mathbb{R}^{2}\right)$ which are $Y$-periodic. This space is naturally endowed with a Hilbert space structure.

Probability space. A general event $\omega \in \Omega$ will be represented as

$$
\omega=\left(\left(\theta_{j}, \rho_{j}, \varepsilon_{j}\right)_{j \in \mathbb{Z}^{2}}, y\right)=(m, y),
$$

where $m=\left(m_{j}\right)_{j \in \mathbb{Z}^{2}}$ is a sequence of elements in $M$ and $y \in Y$. We consider a reference Borel probability law $\mathbf{p}$ on $M$ which, for the sake of simplicity, we assume to be compactly supported. Then our general probability space $(\Omega, \mathcal{A}, \mathbb{P})$ reads

$$
\Omega:=\Pi \times Y, \quad \mathcal{A}=\mathcal{F} \otimes \mathscr{B}(Y), \quad \mathbb{P}=\boldsymbol{\pi} \otimes \mathcal{L}^{2},
$$

being $\mathcal{L}^{2}$ the Lebesgue measure and the space $\Pi:=M^{\mathbb{Z}^{2}}$ (metrisable and compact) endowed with the product measure $\boldsymbol{\pi}=\bigotimes_{\mathbb{Z}^{2}} \mathbf{p}$ and Borel tribe $\mathcal{F}=\bigotimes_{\mathbb{Z}^{2}} \mathscr{B}(M)$.

In the following $C(\Omega)$ will denote the space of bounded continous functions on $\Omega$. The expectation of a random function $f(\omega)$ in $L^{1}(\Omega, \mathbb{P}, \mathcal{A})$ is denoted by the symbol $\mathbb{E}(f)$.

We indicate in bold symbol $\boldsymbol{\theta}_{j}(\omega)$ (respectively $\boldsymbol{\rho}_{j}(\omega), \boldsymbol{\varepsilon}_{j}(\omega), \boldsymbol{y}(\omega)$ ) the random variable associated with the projection $\omega \in \Omega \mapsto \theta_{j}$ (resp. $\omega \mapsto \rho_{j}, \varepsilon_{j}, y$ ). The triples $\left(\boldsymbol{\theta}_{j}(\omega), \boldsymbol{\rho}_{j}(\omega), \boldsymbol{\varepsilon}_{j}(\omega)\right.$ ) are independent and identically distributed random variables, which seen as functions on $M$ share the given probability law $\mathbf{p}$. Accordingly the random disk $\mathcal{D}_{\eta}^{j}(\omega)$ is given by (1.2).

Next we introduce the following Borel subsets of $\Omega$ :

$$
\Sigma=\left\{\omega \in \Omega:\left|y-\theta_{0}\right|<\rho_{0}\right\}, \quad \Sigma^{*}=\Omega \backslash \bar{\Sigma} .
$$

We notice that the real number $\mathbb{P}(\Sigma) \in(0,1)$ represents the average filling ratio of the inclusions in $\mathcal{B}$. Indeed, recalling that the last marginal of $\mathbb{P}$ coincides with the Lebesgue measure on $Y$, by the law of large numbers it holds almost surely:

$$
\lim _{\eta \rightarrow 0} \frac{\left|\mathcal{D}_{\eta}(\omega)\right|}{|\mathcal{B}|}=\mathbb{P}(\Sigma)=\pi \int_{M} \rho^{2} \mathbf{p}(\mathrm{d} \theta \mathrm{d} \rho \mathrm{d} \varepsilon)
$$


Remark 2.1. If $(\theta, \rho, \varepsilon)$ belongs to $M$ then the disk $B(\theta, \rho)$ is contained in $Y$ and its distance to the boundary $\partial Y$ is at least $\delta$. The prescription of such constant $\delta>0$ will be crucial in order to obtain a uniform $L^{2}(\mathcal{B})$ estimate for the solution $u_{\eta}$ and its convergence in a suitably strong sense (in particular $\delta>0$ is required in order to obtain Lemma A.2 which is a key tool for proving Propositions 6.6 and 6.9).

Effective permittivity. The effective permittivity tensor $\varepsilon^{\text {eff }}$ that arises from our asymptotic analysis turns out to be symmetric positive and independent of the frequency. It relies only on the geometry of the rods, namely on the distribution law of their radius and centers. In fact the computation of $\varepsilon^{\text {eff }}$ falls in the homogenization theory for Neumann problems on random perforated sets as developed for instance in [17]. We have the following variational characterization, for every $z \in \mathbb{R}^{2}$

$$
\varepsilon^{\mathrm{eff}} z \cdot z:=\inf \left\{\int_{\Omega}|\sigma|^{2} \mathrm{~d} \mathbb{P}: \sigma \in L_{\mathrm{sol}}^{2}\left(\Omega ; \mathbb{R}^{2}\right), \sigma=0 \text { in } \Sigma, \mathbb{E}(\sigma)=z\right\}
$$

where $L_{\text {sol }}^{2}\left(\Omega ; \mathbb{R}^{2}\right)$ denotes the set of solenoid random vector fields as defined in (4.10). The minimum above is taken over a closed subspace of $\left(L^{2}(\Omega)\right)^{2}$ which roughly corresponds to vector fields on $\mathbb{R}^{2}$ which are divergence free and vanish on the random set $\mathcal{D}(\omega)$ (see Prop. 4.6). It is easy to check that, for every $z=\left(z_{1}, z_{2}\right) \in \mathbb{R}^{2}$, the solution $\sigma$ to (2.3) exists and is unique. By linearity, it is given by $\sigma=z_{1} \sigma^{1}+z_{2} \sigma^{2}$ where, for $i=1,2, \sigma^{i}$ denotes the solution associated with $z=e_{i}$. Therefore, the effective tensor $\varepsilon^{\text {eff }}$ can be witten as follows

$$
\varepsilon_{i, j}^{\mathrm{eff}}=\mathbb{E}\left(\sigma^{i} \cdot \sigma^{j}\right)
$$

Let us mention some straightforward estimates for that tensor. Firstly we observe that, if we set $Y_{\delta}:=\{y \in$ $Y: \operatorname{dist}(y, \partial Y)>\delta\}$, then by (2.1) and (2.2) it holds $\Sigma \subset \Pi \times Y_{\delta}$ and therefore vector fields $\sigma=\sigma(y)$ are admissible if they belong to the class $\mathcal{S}_{\delta}$ of restrictions to $Y$ of $Y$-periodic, divergence free fields of $L_{\text {loc }}^{2}\left(\mathbb{R}^{2}\right)$ vanishing in $Y_{\delta}$ (see (ii) of Prop. 4.6). Therefore the infimum in (2.3) is finite and attained in a unique $\sigma$. Futhermore the following upper bound holds:

$$
\varepsilon^{\text {eff }} \leq C(\delta) \mathrm{I}_{2} \quad \text { with } \quad C(\delta):=\inf \left\{\int_{Y}|\sigma|^{2} \mathrm{~d} y: \sigma \in \mathcal{S}_{\delta}, \int_{Y} \sigma(y)=e\right\},
$$

where $e$ is an arbitrary unit vector and $\mathrm{I}_{2}$ denotes the two by two identity matrix. Note that the constant $C(\delta)$ represents the inverse of the effective diffusion coefficient in the (determinist) case of a periodic perforated domains with square holes (it blows up to infinity as $\delta \rightarrow 0$ ). On the other hand the effective tensor $\varepsilon^{\text {eff }}$ is non degenerate. In fact as can be easily checked by Young inequality, we have the following lower bound

$$
\varepsilon^{\mathrm{eff}} \geq \frac{1}{\mathbb{P}\left(\Sigma^{*}\right)} \mathrm{I}_{2}
$$

Indeed one has $\int_{\Sigma^{*}}|\sigma|^{2} \geq 2 z \cdot z^{*}-\left|z^{*}\right|^{2} \mathbb{P}\left(\Sigma^{*}\right)$, for every admissible $\sigma$ and $z^{*} \in \mathbb{R}^{2}$, thus (2.6) by taking the supremum in $z^{*}$.

Remark 2.2. Unfortunately the formula (2.3) is far to be explicit and $\varepsilon^{\text {eff }}$ is not necessarily a scalar tensor unless the probability distribution of the center $\boldsymbol{\theta}_{0}(\omega)$ is invariant by $\pi / 2$ rotations. Note that if the radius of the rods is a constant $\rho_{0}$ (i.e. the probability law of $\boldsymbol{\rho}_{0}$ is a Dirac mass) then $\varepsilon^{\text {eff }}$ is scalar, does not depend on the law of the center $\boldsymbol{\theta}_{0}$ and (2.3) agrees with the classical formula of the deterministic case (see [8]).

Effective permeability. The effective permeability $\mu^{\text {eff }}$ depends on the frequency $\nu$ and its behaviour is ruled by the spectral values of the Dirichlet Laplace operator in the two dimensional unit disk $D:=B(0,1)$. Let us denote by $\sigma_{0}:=\left\{\lambda_{n}, n \in \mathbb{N}^{*}\right\}$ with $0<\lambda_{1}<\lambda_{2} \leq \ldots \leq \lambda_{n} \leq \ldots$ the eigenvalues of this operator and let $\left\{\varphi_{n}, n \in \mathbb{N}^{*}\right\}$ be an associated orthonormal basis of real eigenvectors in $L^{2}(D)$. We set

$$
c_{n}:=\int_{D} \varphi_{n} \quad\left(\text { thus } 1=\sum c_{n} \varphi_{n} \text { in } L^{2}(D) \text { and } \sum\left|c_{n}\right|^{2}=\pi\right) .
$$


Then, recalling the definition of $\Sigma$ in (2.2), we introduce the random variable $\Lambda(\omega)$

$$
\Lambda(\omega)= \begin{cases}1+\sum_{n=1}^{+\infty} c_{n} \frac{k_{0}^{2} \varepsilon_{0}(\omega) \boldsymbol{\rho}_{0}^{2}(\omega)}{\lambda_{n}-k_{0}^{2} \varepsilon_{0}(\omega) \boldsymbol{\rho}_{0}^{2}(\omega)} \varphi_{n}\left(\frac{\boldsymbol{y}(\omega)-\boldsymbol{\theta}_{0}(\omega)}{\boldsymbol{\rho}_{0}(\omega)}\right) & \text { if } \omega \in \Sigma \\ 1 & \text { if } \omega \in \Sigma^{*}\end{cases}
$$

As will seen later in Section 5, this function $\Lambda$ solves a stochastic version of the classical Helmholz resonator problem (for which we refer to Lemma A.1 in the Appendix).

The effective permeability $\mu^{\text {eff }}\left(k_{0}\right)$ can be then expressed as a function of the incident wave number $k_{0}$ (recall $\left.k_{0}=\frac{\nu}{c}\right)$ as follows:

$$
\mu^{\mathrm{eff}}\left(k_{0}\right)=\mathbb{E}(\Lambda(\omega))=1+\sum_{n=1}^{+\infty} c_{n}^{2} \int_{M} \frac{\varepsilon \rho^{4} k_{0}^{2}}{\lambda_{n}-\varepsilon \rho^{2} k_{0}^{2}} \mathrm{~d} \mathbf{p}(\theta, \rho, \varepsilon) .
$$

We notice that the random fluctuations of the center $\theta$ of the disks have no effect on the value of $\mu^{\text {eff }}$ (which depends only on the $(\rho, \varepsilon)$-marginal of $\mathbf{p})$.

Homogenization result. The main result of this paper stated below requires two hypotheses on the probability law p:

$$
\begin{gathered}
\exists r>0 \text { such that } \int_{M}\left[\operatorname{dist}\left(\varepsilon \rho^{2} k_{0}^{2}, \boldsymbol{\sigma}_{0}\right)\right]^{-(2+r)} \mathrm{d} \mathbf{p}<\infty, \\
\mathbf{p}(\{(\theta, \rho, \varepsilon) \in M \mid \Im(\varepsilon)>0\})>0 .
\end{gathered}
$$

We will establish that, under these assumptions, for almost all $\tilde{\omega} \in \Omega$ the magnetic field $u_{\eta}(\cdot, \tilde{\omega})$ characterized by equations (1.4) and (1.6) does converge pointwise outside the obstacle to a non random function $u \in W_{\text {loc }}^{1,2}\left(\mathbb{R}^{2}\right)$ characterized as the unique solution to

$$
\left\{\begin{array}{l}
\operatorname{div}(\mathbf{A}(x) \nabla u)+k_{0}^{2} \mathbf{b} u=0, \\
u-u^{\text {inc }} \quad \text { satisfies the outgoing radiation condition (1.6). }
\end{array}\right.
$$

Here $\mathbf{A}(x)$ and $\mathbf{b}(x)$ represent the the local permittance and permeability functions over all $\mathbb{R}^{2}$ defined by:

$$
\begin{aligned}
& \mathbf{A}(x):=1_{\mathcal{B}}(x)\left(\varepsilon^{\text {eff }}\right)^{-1}+1_{\mathbb{R}^{2} \backslash \mathcal{B}}(x) I_{2}, \\
& \mathbf{b}(x):=1_{\mathcal{B}}(x) \mu^{\text {eff }}\left(k_{0}\right)+1_{\mathbb{R}^{2} \backslash \mathcal{B}}(x),
\end{aligned}
$$

being the effective permittivity tensor given by $\varepsilon^{\text {eff }}$ and the frequency dependent permeability $\mu^{\text {eff }}\left(k_{0}\right)\left(k_{0}=\right.$ $\left.\omega \sqrt{\varepsilon_{0} \mu_{0}}\right)$ by expansion $(2.8)$.

Let us emphasize that the first equation in (2.11) has to be understood in the distributional sense. In particular the fact that $\nabla u, \operatorname{div}(\mathbf{A}(x) \nabla u)$ belong to $L_{\text {loc }}^{2}$ imply the following transmission conditions on the boundary of $\mathcal{B}$ :

$$
u^{+}=u^{-}, \quad \partial_{n} u^{+}=\left(\left(\varepsilon^{\mathrm{eff}}\right)^{-1} \nabla u^{-}\right) \cdot n,
$$

where superscript \pm indicate the traces from outside and inside for $u$ and the normal derivative $\partial_{n} u$ (being $n$ the exterior normal on $\partial \mathcal{B}$ ). These transmission conditions indicate that $u$ fits with the limit magnetic field seen from the exterior. We wish however to point out that inside the obstacle $u(x)$ differs from the weak limit of $u_{\eta}$. In order to account the fast oscillating behavior of $u_{\eta}$ inside $\mathcal{B}$, we will use the random function $\Lambda(\omega)$ given by (2.7) and define

$$
u_{0}(x, \omega):=u(x)\left(\Lambda(\omega) 1_{\mathcal{B}}(x)+1_{\mathbb{R}^{2} \backslash \mathcal{B}}(x)\right) .
$$


Theorem 2.3. Assume that the distribution of rods is given by the i.i.d random variables $\left(\theta_{j}, \rho_{j}, \varepsilon_{j}\right)$ with a probability law p satisfying conditions (2.9) and (2.10).

Then the diffraction problem (2.11) admits a unique solution $u$ and, for $\mathbb{P}$-almost all $\tilde{\omega} \in \Omega$, there holds

$$
u_{\eta}(\cdot, \tilde{\omega}) \rightarrow \mathbf{b} u \quad \text { weakly in } L_{\mathrm{loc}}^{2}\left(\mathbb{R}^{2}\right) .
$$

Furthermore, on compact subsets of $\mathbb{R}^{2} \backslash \mathcal{B}$ (where $\mathbf{b}=1$ ), the convergence $u_{\eta}(\cdot, \tilde{\omega}) \rightarrow u$ is uniform as well as for all derivatives. Eventually the defect of strong $L^{2}$-convergence in $\mathcal{B}$ can be recast from the following equalities, holding for every bounded Borel subset $A \subset \mathbb{R}^{2}$ :

$$
\lim _{\eta \rightarrow 0} \int_{A}\left|u_{\eta}(x, \tilde{\omega})-\mathbf{b} u\right|^{2} \mathrm{~d} x=\mathbb{E}\left(\int_{A}\left|u_{0}(x, \cdot)-\mathbf{b} u\right|^{2} \mathrm{~d} x\right)=\operatorname{var}(\Lambda) \int_{\mathcal{B} \cap A}|u(x)|^{2} \mathrm{~d} x
$$

being $u_{0}$ given by $(2.14)$ and $\operatorname{var}(\Lambda):=\mathbb{E}\left(|\Lambda|^{2}\right)-|\mathbb{E}(\Lambda)|^{2}$ the variance of $\Lambda$.

The proof of Theorem 2.3 is quite long and involved. It is postponed in Section 6 where the arguments are presented along three steps. The most delicate issue is the $L^{2}$ upper bound estimate (see (6.1)) which is proved a posteriori in the last step by using a contradiction argument (in the same line as in $[4,5]$ ). Previous to this proof, in Section 5, we assume a priori this $L^{2}$ - upper bound in order to anticipate the two-scale analyis of the system and identify directly the stochastic two-scale limits of the relevant quantities. In order to lighten the presentation, some technical results are posponed in the Appendix (Sect. A).

Remark 2.4. This result generalizes to the random case the results of [3]. More precisely, the periodic case is recovered by taking the probability $\mathbf{p}$ to be a Dirac mass at $\left(\boldsymbol{\theta}_{0}, \boldsymbol{\rho}_{0}, \boldsymbol{\varepsilon}_{0}\right)$. Conditions (2.9) and (2.10) are trivially satisfied provided $\Im\left(\varepsilon_{0}\right)>0$. In this case the probability space $(\Omega, \mathbb{P})$ can be identified with $\left(Y, \mathcal{L}^{2}\right)$ and the function $u_{0}=u_{0}(x, y) \in L_{\mathrm{loc}}^{2}\left(\mathbb{R}^{2} ; W_{\sharp}^{1,2}(Y)\right)$ coincides with the classical two-scale limit of $u_{\eta}$ (see [1]). Furthermore, the convergence in (2.15) yields the strong two-scale convergence of $u_{\eta}$ to $u_{0}(x, y)$ in $\mathcal{B}$ and the strong $L^{2}$ convergence $u_{\eta} \rightarrow u$ on every bounded subset $A$ such that $\mathcal{B} \cap A$ is Lebesgue negligible. This is an improvement of the result presented in [3]. In fact this improvement of the convergence is extended here to the random setting by using the dynamical system approach developed in Section 4 and the map $T$ defined in (4.1). More precisely (see Prop. 6.6), we will show that for any ball $B_{R}$ of arbitrary large radius, there holds for almost all $\tilde{\omega} \in \tilde{\Omega}$

$$
\lim _{\eta \rightarrow 0^{+}} \int_{B_{R}}\left|u_{\eta}(x, \tilde{\omega})-u_{0}\left(x, T_{\frac{x}{\eta}} \tilde{\omega}\right)\right|^{2} \mathrm{~d} x=0 .
$$

Remark 2.5. We can see easily that the imaginary part of $\mu^{\mathrm{eff}}\left(k_{0}\right)$ is positive if the condition (2.10) is verified. Thanks to this assumption, the limit problem (2.11) turns out to be well posed. Notice that (2.9) and (2.10) are trivially satisfied if the support of the permittivity law in the rods does not intersect the real axis. If it is not the case, it looks natural to add a small dissipative parameter so that our homogenization result applies and then to send this parameter to zero. This important issue is discussed in Section 3.

\section{Frequency Dependent efFective Permeability LaW}

In the context of finding new metamaterials with magnetic activity, the homogenization result in Theorem 2.3 demonstrates that random dielectric structures are theoretically able to produce permeability laws like in (2.8). In particular effective permeability coefficients $\mu^{\mathrm{eff}}$ with large negative real part are possible in suitable ranges of frequencies. At this stage, it is worth to study the behaviour of the effective permeability $\mu^{\text {eff }}\left(k_{0}\right)$ with repect to the probability law $\mathbf{p}$ characterizing the random rods. 

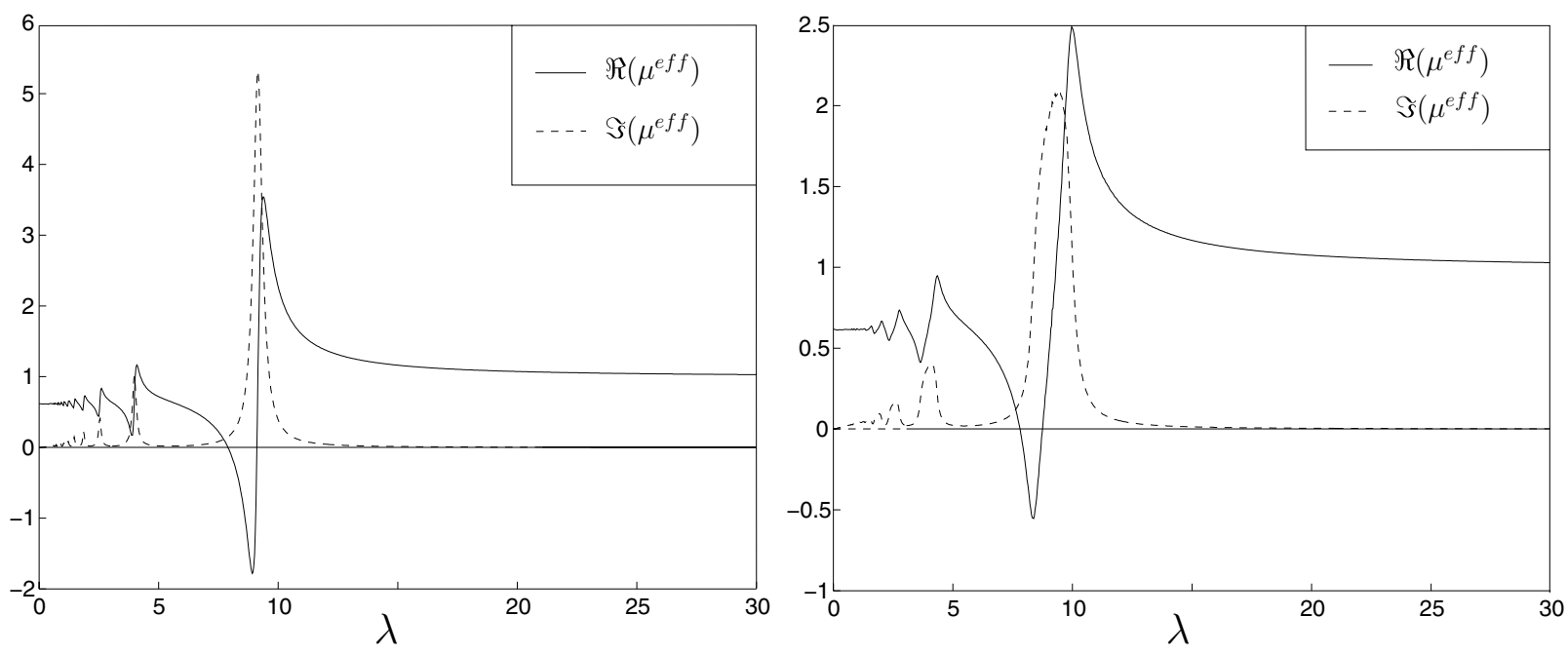

FiguRe 2. Resonances in determinist (left) and random (right) cases.

\subsection{Resonant effects}

Let us rewrite (2.8) as

$$
\mu^{\mathrm{eff}}\left(k_{0}\right)=1+\sum_{n \in I} c_{n}^{2} \int_{M} \frac{\rho^{2} k_{0}^{2}}{k_{n}^{2}(\rho, \varepsilon)-k_{0}^{2}} \mathrm{~d} \mathbf{p}(\theta, \rho, \varepsilon),
$$

being $I:=\left\{n \geq 1: c_{n} \neq 0\right\}$ and $k_{n}:=k_{n}(\rho, \varepsilon) \in \mathbb{C}^{+}$such that $k_{n}^{2}=\frac{\lambda_{n}}{\varepsilon \rho^{2}}$.

The internal resonant frequencies are characterized by the real random numbers $\left\{\nu_{n}, n \in I\right\}$ where $\nu_{n}=$ $\sqrt{\Re e\left(k_{n}^{2}\right)}$. Indeed, if the random variable $\Im(\varepsilon)$ remains small, a huge variation of $\mu^{\text {eff }}$ will appear when $k_{0}$ passes through one of the $\nu_{n}$ 's with a change of sign of the real part. On the other hand it is clear that the random fluctuations of the $\nu_{n}$ 's contribute to damp the leading term in the power series.

To illustrate these facts, we have considered rods with constant relative permittivity $\varepsilon_{r}=100+5 i$ and compared the situations where the reference radius $\rho_{0}=0.375$ is kept constant or is uniformly distributed on interval $I:=[0.3,0.45]$. The first case is purely determinist and corresponds to taking $\mathbf{p}$ to be a Dirac mass at $\left(\rho_{0}, \theta_{0}, \varepsilon_{r}\right)$ (the position of center $\theta_{0}$ has no influence) whereas in the second case we have $\mathbf{p}=1_{\mathrm{I}}(\rho) \mathrm{d} \rho \otimes \delta(\theta-$ $\left.\theta_{0}\right) \otimes \delta\left(\varepsilon-\varepsilon_{r}\right)$.

In Figure 2, we have drawn the real and imaginary parts of $\mu^{\text {eff }}$ predicted by the homogenization formula in the determinist case (left hand side) and in the random case respectively (right hand side). The abscissa $\lambda=\frac{2 \pi}{k_{0}}$ represents the normalized wavelength.

\subsection{The case of vanishingly small loss dielectric}

We discuss now about the dependence of the effective problem with respect to the permittivity law, in particular in the case where this law $\mathbf{p}$ is supported on the real axes (non dissipative medium) possibly violating the condition (2.9) needed for the validity of our homogenization result. For the sake of simplicity we take $\mathbf{p}$ of the form

$$
\mathbf{p}(\theta, \rho, \varepsilon)=\delta_{0}\left(\theta-\theta_{0}\right) \otimes \gamma(\rho) \otimes g(a) \mathrm{d} a \otimes \delta_{0}(b),
$$

where $\varepsilon=a+i b, g$ is a Lipschitz function on $\mathbb{R}$ with compact support and $\gamma$ is an arbitrary probability law for $\rho$ on $\left(0, \rho^{+}\right]$(such that $B\left(\theta_{0}, \rho^{+}\right) \subset \subset Y$ ). Then we introduce a small absorption parameter $h>0$ (designed to 

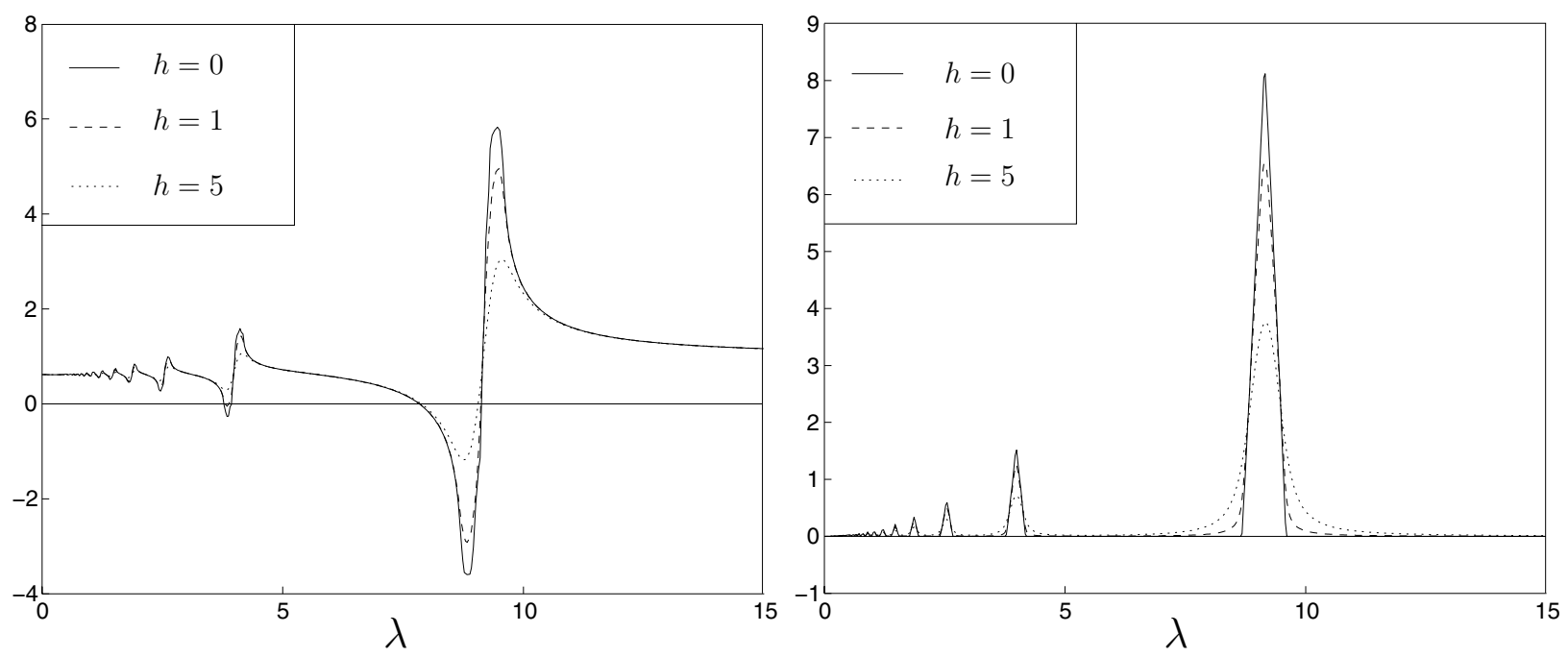

FiguRE 3. Real and imaginary part of $\mu_{h}^{\text {eff }}$ for $h \in\{0,1,5\}$.

tend to zero) and set

$$
\mathbf{p}_{h}:=\delta_{0}\left(\theta-\theta_{0}\right) \otimes \gamma(\rho) \otimes g(a) \mathrm{d} a \otimes \delta_{0}(b-h) .
$$

Clearly, for every $h>0$ the condition (2.9) holds and $\mathbf{p}_{h} \stackrel{*}{\rightarrow} \mathbf{p}$ as $h$ tends to zero. For every $h>0$, the condition (2.10) holds and by applying Theorem 2.3, the homogenized medium obtained in the limit $\eta \rightarrow 0$ is characterized by the effective permeability

$$
\mu_{h}^{\mathrm{eff}}\left(k_{0}\right):=1+\sum_{n}\left|c_{n}\right|^{2} I_{h, n}, \quad I_{h, n}:=\int_{M} \frac{\varepsilon k_{0}^{2} \rho^{4}}{\lambda_{n}-\varepsilon k_{0}^{2} \rho^{2}} p_{h}(\mathrm{~d} \theta \mathrm{d} \rho \mathrm{d} \varepsilon) .
$$

Our goal is to propose an effective theory for non dissipative random structures, like those described by (3.1), by passing to the limit $h \rightarrow 0$. For every $n \in \mathbb{N}$, we introduce the real compactly supported Lipschitz function $f_{n}: \mathbb{R} \mapsto \mathbb{R}_{+}$defined by

$$
f_{n}(s):=\frac{\lambda_{n}}{k_{0}^{2}} \int_{\left(0, \rho^{+}\right]} g\left(\frac{\lambda_{n}}{k_{0}^{2} \rho^{2}}+s\right) \gamma(\mathrm{d} \rho) .
$$

Proposition 3.1. For every $k_{0} \in \mathbb{R}^{+}$, there holds $\mu_{h}^{\mathrm{eff}}\left(k_{0}\right) \rightarrow \mu^{\mathrm{eff}}\left(k_{0}\right)$ where

$$
\mu_{0}^{\mathrm{eff}}\left(k_{0}\right)=1-\pi \int_{(0, \rho+]} \rho^{2} \gamma(\mathrm{d} \rho)+\sum_{n \in \mathbb{N}}\left|c_{n}\right|^{2}\left(-\mathrm{PV} \int \frac{f_{n}(s)}{s} \mathrm{~d} s+i \pi f_{n}(0)\right),
$$

(where $\operatorname{PV}\left(\int f\right)=\lim _{s \rightarrow 0} \int_{\mathbb{R} \backslash[-s, s]} f(t) \mathrm{d} t$ and refers to the Cauchy principal value).

Some comments about the validity of the resulting model can be found in Remark 3.2. In order to illustrate the random dependance, we draw in Figure 3 the function $\mu_{h}^{\text {eff }}$ for decreasing values of parameter $h$ and $\mu_{0}^{\text {eff }}$ given in (3.5) which corresponds to $h=0$. To simplify, we choosed the law of $\gamma(\rho)$ to be Dirac mass at $\rho_{0}=0.35$ while $g(a)=10^{-2}(10-|a-100|)^{+}$.

Remark 3.2. The limit absorption process described in Proposition 3.1 is stable in the sense that the result remains unchanged if in $(3.2)$ we substitute $\delta_{0}(b-h)$ with $\frac{1}{h} \zeta\left(\frac{b}{h}\right)$, being $\zeta$ any probability law on $\mathbb{R}_{*}^{+}$compatible 
with (2.9). At this point it is tempting to claim that, even if the initial random law in the rods is non dissipative, the limit medium as $\eta \rightarrow 0$ can still be described by the homogenization theory with an effective permeability law given by (3.5).

However the situation is not so simple and we face here a paradox. Indeed, if the initial probability law is non dissipative like in (3.1), it looks obvious that the homogenized limit should be described by real effective coefficients. Surprisingly it is not the case in our model: according to formula (3.5) the imaginary part of $\mu_{0}^{\text {eff }}$ shows positive values when the incident wave number $k_{0}$ ranges in suitable intervals of $\mathbb{R}^{+}$. Several possible explanations can be addressed. First our asymptotic analysis $\eta \rightarrow 0$ has been performed in harmonic regime fixing a priori the frequency of the incident wave. Second we applied the limit absorption principle $(h \rightarrow 0)$ once the microscale parameter $\eta$ was sent to zero. This clearly is only one strategy among many others.

Remark 3.3. If $\Im\left(\mu_{0}^{\text {eff }}\left(k_{0}\right)\right)>0$, Proposition 3.1 can be improved as follows: let $u_{h}$ be the solution of $(2.11)$ being in (2.12) $\mu^{\mathrm{eff}}$ substituted with $\mu_{h}^{\mathrm{eff}}$. Then $u_{h} \rightarrow u$ strongly in $L_{\text {loc }}^{2}\left(\mathbb{R}^{2}\right)$ where $u$ is the unique solution of (2.11) with $\mu_{0}^{\text {eff }}$ in (2.12) determined by (3.5) (the main point of the proof is an $L^{2}$ uniform estimate for $\left\{u_{h}\right\}$ which can be obtained by using the same kind of contradiction argument as in the proof of Theorem 2.3).

Proof of proposition 3.1. Let us plug in (3.3) the expression (3.2) of $p_{h}$. By using the change of variable $s=a-\frac{\lambda_{n}}{k_{0}^{2} \rho^{2}}$ together with Fubini's formula, we are led to

$$
I_{h, n}=\int_{(0, \rho+]} \rho^{2} \gamma(\mathrm{d} \rho)-\int_{-\infty}^{+\infty} \frac{s}{s^{2}+h^{2}} f_{n}(s) \mathrm{d} s+i \int_{-\infty}^{+\infty} \frac{h}{s^{2}+h^{2}} f_{n}(s) \mathrm{d} s .
$$

Here $f_{n}$ given by (3.4) inherits the Lipschitz continuity of $g$, and therefore, for every $n$, there holds:

$$
\begin{gathered}
\lim _{h \rightarrow 0} \int_{-\infty}^{+\infty} \frac{s}{s^{2}+h^{2}} f_{n}(s) \mathrm{d} s=\operatorname{PV} \int \frac{f_{n}(s)}{s} \mathrm{~d} s \\
\lim _{h \rightarrow 0} \int_{-\infty}^{+\infty} \frac{h}{s^{2}+h^{2}} f_{n}(s) \mathrm{d} s=\pi f_{n}(0) .
\end{gathered}
$$

Thus we have

$$
\lim _{h \rightarrow 0} I_{h, n}=I_{n}:=\int_{(0, \rho+]} \rho^{2} \gamma(\mathrm{d} \rho)-\mathrm{PV} \int \frac{f_{n}(s)}{s} \mathrm{~d} s+i \pi f_{n}(0) .
$$

In view of (3.5), (3.7) and recalling that $\sum_{n}\left|c_{n}\right|^{2}=\pi$, the convergence (3.5) is established provided we have $\lim _{h \rightarrow 0}\left(\sum_{n}\left|c_{n}\right|^{2} I_{h, n}\right)=\sum_{n}\left|c_{n}\right|^{2} I_{n}$. In order to prove previous equality as well as the summability of the series, it is enough to show the following uniform upper bound for suitably large $n_{0}$ :

$$
\sup \left\{\left|I_{h, n}\right|: n \geq n_{0}, h>0\right\}<+\infty .
$$

Let us set $\alpha_{n}:=\inf \left\{|s|: f_{n}(s) \neq 0\right\}$. Looking at (3.4), since $\rho$ ranges in $[0,1 / 2)$ and $g$ is compactly supported while $\lambda_{n} \rightarrow \infty$ as $n \rightarrow \infty$, it is easy to check that

$$
\liminf _{n \rightarrow \infty} \frac{\alpha_{n}}{\lambda_{n}} \geq \frac{4}{k_{0}^{2}}
$$

On the other hand, from the expression in (3.6), we deduce that

$$
\left|I_{h, n}\right| \leq \frac{1}{4}+\frac{1}{\sqrt{\alpha_{n}^{2}+h^{2}}} \int f_{n}(s) \mathrm{d} s \leq \frac{1}{4}+\frac{\lambda_{n}}{k_{0}^{2} \alpha_{n}} .
$$

The upper bound (3.8) follows and the proof is finished. 


\section{Stochastic FRAMEWORK}

The random set $\mathcal{D}_{\eta}(\omega)$ defined in (1.2) can be generated (after a proper rescaling) through a continuous dynamical system. To that aim we will follow basically the approach developed in [17] (for the homogenization of randomly perforated domains) and more recently in [26]. We begin this Section by some classical background on the dynamical system approach. Then we specialize in the construction of stochastic Sobolev spaces with an emphasize on the characterization obtained in Proposition 4.4 (which improves some tools given in [26]). Pratical consequences for our particular case are given in Proposition 4.6.

Dynamical system and ergodicity. On our probability space $(\Omega, \mathcal{A}, \mathbb{P})$, we define a group of transformations $T_{x}: \Omega \rightarrow \Omega, x \in \mathbb{R}^{2}$ as follows

$$
T_{x}\left(\left(m_{j}\right)_{j \in \mathbb{Z}^{2}}, y\right)=\left(\left(m_{j+[x+y]}\right)_{j \in \mathbb{Z}^{2}}, x+y-[x+y]\right),
$$

where by abuse of notations we write $[x]$, for $x \in \mathbb{R}^{2}$, meaning the couple $\left(\left[x_{1}\right],\left[x_{2}\right]\right)$ formed by the integer parts of the coordinates of $x$.

It is easy to check that the map $(x, \omega) \mapsto T_{x}(\omega)$ is Borel regular and that it enjoys the usual properties of a ergodic dynamical system: for every $x \in \mathbb{R}^{2}, T_{x}$ preserves the measure $\mathbb{P}$. Moreover since the discrete dynamical system associated with the Bernoulli shift over the product space $(\Pi, \boldsymbol{\pi}):=\left(M^{\mathbb{Z}^{2}}, \bigotimes_{\mathbb{Z}^{2}} \mathbf{p}\right)$ is ergodic (see for instance [12], Thm. 8.4.5), it can be easily checked (as noticed in [26]) that $T_{x}$ is ergodic on $(\Omega, \mathcal{A}, \mathbb{P}$ ). This means that, for every $x \in \mathbb{R}^{2}$, one has $\mathbb{P}(A) \in\{0,1\}$ for every $T_{x}$-invariant $A \in \mathcal{A}$ (i.e. such that $\left.\mathbb{P}\left(\left(T_{x} A \backslash A\right) \cup\left(A \backslash T_{x} A\right)\right)=0\right)$.

As a fundamental consequence, by Birkhoff ergodic theorem (see [10], Prop. 12.2.II), for every $f \in L^{1}(\Omega, \mathcal{A}, \mathbb{P})$, the following equality holds almost surely

$$
\lim _{r \rightarrow \infty} \frac{1}{\pi r^{2}} \int_{B_{r}} f\left(T_{x} \omega\right) \mathrm{d} x=\int_{\Omega} f d \mathbb{P}, \quad \mathbb{P}-\text { a.s. }
$$

Statistically homogeneous random fields and scaling. With our dynamical system, we associate the family of statistically homogeneous random fields which are functions $f(x, \omega)$ of the kind $F\left(T_{x} \omega\right)$, being $F$ a measurable function on $\Omega$. The rescaled versions $F\left(T_{\frac{x}{n}} \omega\right)$ can be seen as a stochastic counterpart of the class of periodic functions oscillating at scale $\eta$. Such random fields are well fitted to represent our random permittivity because the properties of a rescaled cell $Y_{\eta}^{j}(\omega)=\eta(j-\boldsymbol{y}(\omega)+Y)$ in the configuration $\omega$ can be expressed in terms of the reference cell $Y$ in the configuration $T_{\frac{x}{\eta}} \omega$. More precisely, recalling the definition (2.2) of $\Sigma$, the random sets $\mathcal{D}_{\eta}^{j}(\omega)$ defined in (1.2) can be characterized as follows

$$
x \in \mathcal{D}_{\eta}^{j}(\omega) \Longleftrightarrow j=\left[\frac{x}{\eta}+\boldsymbol{y}(\omega)\right] \text { and } T_{\frac{x}{\eta}} \omega \in \Sigma .
$$

In particular the perforation $\mathcal{D}_{\eta}(\omega)$ introduced in (1.2) satisfies

$$
x \in \mathcal{D}_{\eta}(\omega) \Longleftrightarrow\left(x, T_{\frac{x}{\eta}} \omega\right) \in \mathcal{B}_{\eta}(\omega) \times \Sigma,
$$

with $\mathcal{B}_{\eta}(\omega):=\bigcup_{j \in J_{\eta}(\omega)} \eta(j-\boldsymbol{y}(\omega)+Y)$ and $J_{\eta}(\omega)$ given in (1.3).

Accordingly, since $\varepsilon_{j}(\omega)=\varepsilon_{0}\left(T_{\frac{x}{\eta}} \omega\right)$ on each $D_{\eta}^{j}(\omega)$, the inverse of the random relative permittivity $a_{\eta}(x, \omega)$ reads:

$$
a_{\eta}(x, \omega)=1_{\mathcal{B}_{\eta}(\omega)}(x)\left(\frac{\eta^{2}}{\varepsilon_{0}\left(T_{\frac{x}{\eta}} \omega\right)} 1_{\Sigma}\left(T_{\frac{x}{\eta}} \omega\right)+1_{\Sigma^{*}}\left(T_{\frac{x}{\eta}} \omega\right)\right)+1_{\mathbb{R}^{2} \backslash \mathcal{B}_{\eta}(\omega)}(x) .
$$

Stochastic derivative. We associate with $T_{x}$ a group of transformations $U_{x}, x \in \mathbb{R}^{2}$ acting on measurable functions on $\Omega$, i.e. if $f: \Omega \rightarrow \mathbb{R}$ is $\mathcal{A}$-measurable, then

$$
\left(U_{x} f\right)(\omega)=f\left(T_{x} \omega\right), \quad \omega \in \Omega, \quad x \in \mathbb{R}^{2} .
$$

The following result is classical (see for instance [17], Sect. 7.3) 
Lemma 4.1. For every $x \in \mathbb{R}^{2}, U_{x}$ is a unitary transform on $L^{2}(\Omega, \mathbb{P})$ and the group $x \in \mathbb{R}^{2} \mapsto U_{x}$ is strongly continuous.

The following notion of stochastic derivative is taken from [26].

Definition 4.2 (stochastic partial derivative). The stochastic partial derivative $\partial_{i}^{s}(i=1,2)$ is the infinitesimal generator of the strongly continuous semigroup $U_{t e_{i}}, t \geq 0$ in the space $L^{2}(\Omega)$. For $i=1$, 2 , the domain $D\left(\partial_{i}^{s}\right) \subset L^{2}(\Omega)$ of $\partial_{i}^{s}$ is given by

$$
D\left(\partial_{i}^{s}\right)=\left\{f \in L^{2}(\Omega ; \mathbb{P}): \frac{U_{t e_{i}} f-f}{t} \text { converges in } L^{2}(\Omega ; \mathbb{P}) \text { as } t \rightarrow 0\right\}
$$

and for $f \in D\left(\partial_{i}^{s}\right)$, we set $\partial_{i}^{s} f(\omega)=\lim _{t \rightarrow 0} \frac{U_{t e_{i}} f(\omega)-f(\omega)}{t}, \omega \in \Omega$.

Accordingly the stochastic gradient $\nabla^{\mathrm{s}}: D\left(\nabla^{\mathrm{s}}\right) \rightarrow\left(L^{2}(\Omega ; \mathbb{P})\right)^{2}$ is defined by

$$
\nabla^{s} f:=\left(\partial_{1}^{s} f, \partial_{2}^{s} f\right), \quad f \in D\left(\nabla^{\mathrm{s}}\right):=D\left(\partial_{1}^{s}\right) \cap D\left(\partial_{2}^{s}\right) .
$$

Notice that such a gradient has a vanishing average over $\Omega$. Moreover, for $f, g \in D\left(\nabla^{\mathrm{s}}\right)$, one has $\mathbb{E}\left(\partial_{i}^{s} f g\right)=$ $-\mathbb{E}\left(f \partial_{i}^{s} g\right)$.

Approximation. A function in $L^{2}(\Omega)$ can be approximated by elements of $D\left(\nabla^{\mathrm{s}}\right)$ by using the following analogue of the "convolution" procedure. Let $\Psi_{n}(x):=n^{2} \Psi(n x)$ where $\Psi: \mathbb{R}^{2} \rightarrow \mathbb{R}^{+}$is a fixed smooth regularisation kernel with compact support such that $\int_{\mathbb{R}^{2}} \psi=1$. Then for every $f \in L^{2}(\Omega)$, we set

$$
f_{n}(\omega):=\int_{\mathbb{R}^{2}} f\left(T_{x} \omega\right) \Psi_{n}(x) \mathrm{d} x
$$

Lemma 4.3. For every $f \in L^{2}(\Omega), f_{n}$ given in (4.7) belongs to $D\left(\nabla^{\mathrm{s}}\right)$ and converges strongly to $f$ in $L^{2}(\Omega)$. Moreover it holds

$$
\nabla^{s} f_{n}(\omega)=-\int_{\mathbb{R}^{2}} f\left(T_{x} \omega\right) \nabla \Psi_{n}(x) \mathrm{d} x, \quad \mathbb{P} \text { a.e. } \omega \in \Omega
$$

As a consequence the space $D\left(\nabla^{\mathrm{s}}\right)$ is dense in $L^{2}(\Omega)$ and the operator $\nabla^{\mathrm{s}}$ is closable.

By the change of variable $x \mapsto x+t e_{i}$, we have for $t>0$ and $i \in\{1,2\}$

$$
\frac{f_{n}\left(T_{t e_{i}} \omega\right)-f_{n}(\omega)}{t}=\int_{\mathbb{R}^{2}} f\left(T_{x} \omega\right) \frac{\left(\Psi_{n}\left(x-t e_{i}\right)-\Psi_{n}(x)\right)}{t} \mathrm{~d} x
$$

For small $t$, the integral above can be restricted to a compact neighborhood $K$ of the support of $\Psi$. Then, since $\int_{K \times \Omega}\left|f\left(T_{x} \omega\right)\right|^{2} \mathrm{~d} x \mathrm{~d} \mathbb{P}=|K| E\left(|f|^{2}\right)<+\infty$, by Cauchy-Schwartz:

$$
\frac{f_{n}\left(T_{t e_{i}} \omega\right)-f_{n}(\omega)}{t}+\int_{\mathbb{R}^{2}} f\left(T_{x} \omega\right) \frac{\partial \Psi_{n}}{\partial x_{i}} \mathrm{~d} x \rightarrow 0 \quad \text { strongly in } L^{2}(\Omega) .
$$

Thus $f_{n} \in D\left(\nabla^{\mathrm{s}}\right)$ and (4.8) holds. On the other hand it is easy to check that $f_{n} \rightarrow f$ in $L^{2}(\Omega)$. Therefore we have shown that the operator $\nabla^{\mathrm{s}}$ is densily defined. To check that it is closable we consider $u_{n}$ in $D\left(\nabla^{\mathrm{s}}\right)$ such that $u_{n} \rightarrow 0$ and $\nabla^{s} u_{n} \rightarrow \xi$ in $L^{2}(\Omega)$. For every $f \in D\left(\nabla^{\mathrm{s}}\right)$, one has: $\mathbb{E}\left(\xi_{i} f\right)=\lim _{n} \mathbb{E}\left(\partial_{i}^{s} u_{n} f\right)=$ $-\lim _{n} \mathbb{E}\left(u_{n} \partial_{i}^{s} f\right)=0$, thus $\xi_{i}=0$ by the density of $D\left(\nabla^{\mathrm{s}}\right)$.

Stochastic Sobolev space. The Sobolev space $H_{s}^{1}(\Omega)$ will be the completion of $D\left(\nabla^{\mathrm{s}}\right)$ with respect to the Hilbert norm $\|f\|_{H_{s}^{1}}=\|f\|_{L^{2}(\Omega ; \mathbb{P})}+\left\|\nabla^{s} f\right\|_{L^{2}(\Omega ; \mathbb{P})}$. By Lemma 4.3, $D\left(\nabla^{\mathrm{s}}\right)$ is closable and admits a unique 
extension to $H_{s}^{1}(\Omega)$. This extension still denoted $\nabla^{\mathrm{s}}$ acts as a linear continuous operator from $H_{s}^{1}(\Omega)$ into $L^{2}(\Omega)$. We may now define a divergence operator by duality. Let

$$
D\left(\operatorname{div}^{\mathrm{s}}\right):=\left\{f \in\left(L^{2}(\Omega)\right)^{2}: \exists C>0, \mathbb{E}\left(f \cdot \nabla^{s} g\right) \leq C\|g\|_{L^{2}(\Omega)} \quad \forall g \in H_{s}^{1}(\Omega)\right\} .
$$

Then, for every $\sigma \in D\left(\operatorname{div}^{\mathrm{s}}\right)$, the stochastic divergence $\operatorname{div}^{\mathrm{s}} \sigma$ is characterized by the indentity

$$
\mathbb{E}\left(\left(\operatorname{div}^{\mathrm{s}} \sigma\right) g\right)=-\mathbb{E}\left(\sigma \cdot \nabla^{s} g\right) \quad \text { for all } g \in H_{s}^{1}(\Omega) .
$$

Equipped with the norm $\sigma \rightarrow\left[\mathbb{E}\left(|\sigma|^{2}+\left|\operatorname{div}^{\mathrm{s}} \sigma\right|^{2}\right)\right]^{1 / 2}, D\left(\operatorname{div}^{\mathrm{s}}\right)$ is a Hilbert space as well. The subspace $L_{\text {sol }}^{2}(\Omega)$ of "solenoidal" fields will play an important role. It is defined as

$$
L_{\mathrm{sol}}^{2}(\Omega)=\left\{\sigma \in D\left(\operatorname{div}^{\mathrm{s}}\right): \operatorname{div}^{\mathrm{s}} \sigma=0\right\} .
$$

We are now in position to give a characterization of Sobolev space $H_{s}^{1}(\Omega)$ and $D\left(\operatorname{div}^{\mathrm{s}}\right)$.

Proposition 4.4. The following assertions are equivalent:

i) $f \in H_{s}^{1}(\Omega)$

ii) There exists a sequence $f_{n} \in H_{s}^{1}(\Omega)$ such that

$$
f_{n} \rightarrow f \quad \text { in } L^{2}(\Omega) \quad \text { and } \quad \sup _{n} \mathbb{E}\left(\left|\nabla^{s} f_{n}\right|^{2}\right)<+\infty .
$$

iii) There exists a constant $C>0$ such that $\mathbb{E}\left(f \operatorname{div}^{\mathrm{s}} \sigma\right) \leq C\|\sigma\|_{\left(L^{2}(\Omega)\right)^{2}}$, for every $\sigma \in D\left(\operatorname{div}^{\mathrm{s}}\right)$.

iv) For a.a. $\omega$, the realization $x \mapsto f\left(T_{x} \omega\right)$ belongs to $W_{\text {loc }}^{1,2}\left(\mathbb{R}^{2}\right)$ and its distributional gradient $\nabla_{x}\left(f\left(T_{x} \omega\right)\right)$ is a statistically homogeneous random field in $\left(L_{\text {loc }}^{2}\left(\mathbb{R}^{2} \times \Omega\right)\right)^{2}$.

In all these cases, for a.a. $\omega$, we have the following relation holding in the distributional sense in $\mathbb{R}^{2}$ :

$$
\frac{\partial}{\partial x_{i}}\left(f\left(T_{x} \omega\right)\right)=\left(\partial_{i}^{s} f\right)\left(T_{x} \omega\right) \text {. }
$$

Proof. The equivalence between assertions i), ii) and iii) is straightforward once it is observed that the graph $G:=\left\{\left(f, \nabla^{s} f\right) \quad: \quad f \in H_{s}^{1}(\Omega)\right\}$ is a closed subspace of $L^{2}(\Omega) \times\left(L^{2}(\Omega)\right)^{2}$ whose orthogonal reads $G^{\perp}:=$ $\left\{\left(\operatorname{div}^{\mathrm{s}} \sigma, \sigma\right): \sigma \in D\left(\operatorname{div}^{\mathrm{s}}\right)\right\}$.

Let us prove now that every element $f \in H_{s}^{1}(\Omega)$ satisfies the property iv) together with (4.11). In a first step, we assume that $f \in D\left(\nabla^{\mathrm{s}}\right)$. Then for every pair $(\varphi, g) \in C_{c}^{\infty}\left(\mathbb{R}^{2}\right) \times L^{2}(\Omega)$ and $i \in\{1,2\}$, by the invariance property of $\mathbb{P}$, one has

$$
\begin{aligned}
\mathbb{E}\left(g(\omega) \int \partial_{i}^{s} f\left(T_{x} \omega\right) \varphi(x) \mathrm{d} x\right) & =\lim _{t \rightarrow 0} \int_{\Omega} \int_{\mathbb{R}^{2}} \frac{f\left(T_{x+t e_{i}} \omega\right)-f\left(T_{x} \omega\right)}{t} \varphi(x) g(\omega) \mathrm{d} x \mathrm{~d} \mathbb{P} \\
& =\lim _{t \rightarrow 0} \int_{\Omega} \int_{\mathbb{R}^{2}} f\left(T_{x} \omega\right) \frac{\varphi(x)-\varphi\left(x-t e_{i}\right)}{t} g(\omega) \mathrm{d} x \mathrm{~d} \mathbb{P} \\
& =-\int_{\Omega} \int_{\mathbb{R}^{2}} f\left(T_{x} \omega\right) \partial_{x_{i}} \varphi(x) g(\omega) \mathrm{d} x \mathrm{~d} \mathbb{P}
\end{aligned}
$$

from which follows that, a.a. $\omega$, the map $x \mapsto f\left(T_{x} \omega\right)$ belongs to $W_{\text {loc }}^{1,2}\left(\mathbb{R}^{2}\right)$ with $(4.11)$.

In a second step, we consider an approximation sequence $f_{n} \rightarrow f$ in $H_{s}^{1}(\Omega)$ such that $f_{n} \in D\left(\nabla^{\mathrm{s}}\right)$. By the first step, may apply (4.12) to $f_{n}$ which, after the change of variable $\omega \mapsto T_{-x} \omega$, leads to

$$
\mathbb{E}\left(\partial_{i}^{s} f_{n}(\omega) \int_{\mathbb{R}^{2}} \varphi(x) g\left(T_{-x} \omega\right) \mathrm{d} x\right)=-\mathbb{E}\left(f_{n}(\omega) \int_{\mathbb{R}^{2}} \partial_{x_{i}} \varphi(x) g\left(T_{-x} \omega\right) \mathrm{d} x\right) .
$$


The relation above remains true for $f$ by passing to the limit $n \rightarrow \infty$. Then by applying the inverse change of variable $\omega \mapsto T_{-x} \omega$ and by the arbitrariness of $g \in L^{2}(\Omega)$, we conclude that (4.11) holds true for every $f \in H_{s}^{1}(\Omega)$.

Conversely assume that iv) holds and let us prove that $f \in H_{s}^{1}(\Omega)$. We consider the approximation $f_{n}$ defined in (4.7). By Lemma 4.3, we have $f_{n} \in D\left(\nabla^{\mathrm{s}}\right)$ and, since by hypothesis $f\left(T_{x} \omega\right)$ belongs to $W_{\text {loc }}^{1,2}\left(\mathbb{R}^{2}\right)$ we may rewrite (4.8) as $\nabla^{\mathrm{s}} f_{n}(\omega)=\int \nabla_{x}\left[f\left(T_{x} \omega\right)\right] \Psi_{n}(x) \mathrm{d} x$. Then by Jensen inequality (recall $\int \Psi_{n}=1$ ), we derive that

$$
\left|\nabla^{\mathrm{s}} f_{n}(\omega)\right|^{2} \leq \int\left|\nabla_{x}\left[f\left(T_{x} \omega\right)\right]\right|^{2} \Psi_{n}(x) \mathrm{d} x .
$$

Now if $\nabla_{x}\left[f\left(T_{x} \omega\right)\right]$ is statistically homogeneous i.e. of the kind $F\left(T_{x} \omega\right)$ for a suitable $F \in\left(L^{2}(\Omega)\right)^{2}$, we infer that $\sup _{n} \mathbb{E}\left(\left|\nabla^{\mathrm{s}} f_{n}(\omega)\right|^{2}\right) \leq \mathbb{E}\left(|F|^{2}\right)<+\infty$. The conclusion follows by applying assertion ii) to the sequence $\left\{f_{n}\right\}$.

Corollary 4.5. Let $\sigma \in\left(L^{2}(\Omega)\right)^{2}$ and $h \in L^{2}(\Omega)$. Then $\sigma$ belongs to $D\left(\operatorname{div}^{\mathrm{s}}\right)$ with $\operatorname{div}^{\mathrm{s}} \sigma=h$ if and only if, for a.e. $\omega \in \Omega$, the function $x \mapsto \sigma\left(T_{x} \omega\right)$ satisfies $\operatorname{div}_{x}\left(\sigma\left(T_{x} \omega\right)\right)=h\left(T_{x} \omega\right)$ in the distributional sense in $\mathbb{R}^{2}$.

Proof. We set $\sigma_{\varphi}(\omega):=\int_{\mathbb{R}^{2}} \varphi(x) \sigma\left(T_{x} \omega\right) \mathrm{d} x$. for given $\sigma \in\left(L^{2}(\Omega)\right)^{2}$ and $\varphi \in C_{c}^{\infty}\left(\mathbb{R}^{2}\right)$. Then in a similar way as in (4.8), we have that $\sigma_{\varphi} \in D\left(\operatorname{div}^{\mathrm{s}}\right)$ with

$$
\text { for } \mathbb{P} \text { a.e. } \omega \in \Omega, \quad \operatorname{div}^{\mathrm{s}} \sigma_{\varphi}(\omega)=-\int \sigma\left(T_{x} \omega\right) \cdot \nabla \varphi(x) \mathrm{d} x .
$$

Indeed, for every $g \in H_{s}^{1}(\Omega)$, one has $\nabla_{s} g\left(T_{-x} \omega\right)=-\nabla_{x}\left(g\left(T_{-x} \omega\right)\right)$ and by using Fubini and changes of variable $\omega \mapsto T_{ \pm x} \omega$, one gets

$$
\begin{aligned}
\mathbb{E}\left(\sigma_{\varphi} \cdot \nabla^{s} g\right) & =\mathbb{E}\left(\int \sigma\left(T_{x} \omega\right) \cdot \nabla^{s} g(\omega) \varphi(x) \mathrm{d} x\right) \\
& =-\mathbb{E}\left(\int \sigma(\omega) \cdot \nabla_{x}\left(g\left(T_{-x} \omega\right)\right) \varphi(x) \mathrm{d} x\right)=\mathbb{E}\left(\int \sigma(\omega) \cdot \nabla \varphi(x) g\left(T_{-x} \omega\right) \mathrm{d} x\right) \\
& =\mathbb{E}\left(g(\omega) \int \sigma\left(T_{x} \omega\right) \cdot \nabla \varphi(x) \mathrm{d} x\right),
\end{aligned}
$$

which according to (4.9) is equivalent to (4.13).

Let us assume firstly that for a.a $\omega \operatorname{div}_{x}\left(\sigma\left(T_{x} \omega\right)\right)=h\left(T_{x} \omega\right)$ holds in the distributional sense. Then setting $\sigma_{n}:=\sigma_{\Psi_{n}}$ where $\Psi_{n}$ is the regularization kernel used in Lemma 4.3, we infer from (4.13) that $\operatorname{div}^{\mathrm{s}} \sigma_{n}(\omega)=$ $\int h\left(T_{x} \omega\right) \Psi_{n}(x) \mathrm{d} x$. Thus $\left(\sigma_{n}, \operatorname{div}^{\mathrm{s}} \sigma_{n}\right) \rightarrow(\sigma, h)$ in $L^{2}(\Omega)$. As by construction the operator div ${ }^{\mathrm{s}}$ has closed graph, we conclude that $\operatorname{div}^{\mathrm{s}} \sigma=h$.

Assume conversely that $\operatorname{div}^{\mathrm{s}} \sigma=h$ and let $\varphi \in C_{c}^{\infty}\left(\mathbb{R}^{2}\right)$. Then we claim that

$$
\text { for } \mathbb{P} \text { a.e. } \omega \in \Omega, \quad \operatorname{div}^{\mathrm{s}} \sigma_{\varphi}(\omega)=\int h\left(T_{x} \omega\right) \varphi(x) \mathrm{d} x .
$$

Combined with (4.13) and since $\varphi$ is arbitrary, this implies that $\operatorname{div}_{x}\left(\sigma\left(T_{x} \omega\right)\right)$ and $h\left(T_{x} \omega\right)$ agree as distributions. In view of (4.9), we consider an arbitrary $g \in H_{s}^{1}(\Omega)$. Noticing that, for all $x$, $\operatorname{div}^{\mathrm{s}}\left(\sigma\left(T_{x} \omega\right)\right)=$ $\operatorname{div}^{\mathrm{s}} \sigma\left(T_{x} \omega\right)=h\left(T_{x} \omega\right) \quad$ (for a.e. $\omega$ ), we have by using Fubini

$$
\mathbb{E}\left(\sigma_{\varphi} \cdot \nabla^{s} g\right)=\mathbb{E}\left(\int \sigma\left(T_{x} \omega\right) \cdot \nabla^{s} g(\omega) \varphi(x) \mathrm{d} x\right)=-\mathbb{E}\left(g(\omega) \int h\left(T_{x} \omega\right) \cdot \varphi(x) \mathrm{d} x\right) .
$$

The claim (4.14) follows from the characterization (4.9). 
Back to our particular case. Let us particularize previous definitions in the case of the dynamical system introduced in (4.1), where the events $\omega \in \Omega$ are of the form $\omega=(m, y)$ with $(m, y)=\left(\left(m_{k}\right)_{k \in \mathbb{Z}^{2}}, y\right) \in \Pi \times Y$. It is convenient to associate with every $f=f(m, y)$ in $L^{2}(\Omega)$, the following continuation on $\Pi \times \mathbb{R}^{2}$ :

$$
\tilde{f}(m, x):=f\left(\left(m_{k+[x]}\right), x-[x]\right)=f\left(T_{x}(m, 0)\right) .
$$

We observe that, in the case of a function $f$ independent of $m, \tilde{f}$ coincides with the $Y$-periodization of $f$. On the other hand, the realization of a general random field $f$ can be recast from the identity $f\left(T_{x}(m, y)\right)=$ $\tilde{f}(m, x+y)$.

\section{Proposition 4.6.}

i) It holds $f \in H_{s}^{1}(\Omega)$ if and only if $f$ belongs to $L^{2}\left(\Pi, \boldsymbol{\pi} ; W^{1,2}(Y)\right)$ and for $\boldsymbol{\pi}$ almost all $m \in \Pi$, the function $\tilde{f}(m, \cdot)$ is an element of $W_{\text {loc }}^{1,2}\left(\mathbb{R}^{2}\right)$. In this case, one has

$$
\partial_{i}^{s} f(m, \cdot)=\frac{\partial f}{\partial y_{i}}(m, \cdot) \quad \text { a.e in } Y .
$$

ii) A vector field $\sigma \in\left(L^{2}(\Omega)\right)^{2}$ belongs to $L_{\text {sol }}^{2}(\Omega)$ if and only if its extension $\tilde{\sigma}(m, \cdot)$ is divergence free in $\mathbb{R}^{2}$ for almost all $m \in \Pi$.

iii) Let $f \in H_{s}^{1}(\Omega)$ be such that $\nabla^{s} f=0$ for $\mathbb{P}$-almost every $\omega \in \Sigma^{*}$. Then $f$ is constant $\mathbb{P}$-a.e. in $\Sigma^{*}$, that is there exists a contant $c$ (independent of $m$ ) such that for $\boldsymbol{\pi}$ a.a. $m \in \Pi$

$$
f(m, \cdot)=c \quad \text { a.e. on }\left\{y \in Y:\left|y-\boldsymbol{\theta}_{0}(m)\right| \geq \boldsymbol{\rho}_{0}(m)\right\} .
$$

Remark 4.7. According to the assertion i), a function $f(m, y) \in L^{2}(\Omega)$ such that $f=f(y)$ is independent of $m$ belongs to $H_{s}^{1}(\Omega)$ if and only if $f(y) \in W_{\sharp}^{1,2}(Y)$. In the general case, checking that an element $f \in$ $L^{2}\left(\Pi, \boldsymbol{\pi} ; W^{1,2}(Y)\right)$ satisfies $\tilde{f}(m, \cdot) \in W_{\text {loc }}^{1,2}\left(\mathbb{R}^{2}\right)$ amounts to check a compatibility condition for the traces of $f(m, \cdot)$ on $\partial Y$, namely:

$$
f\left(T_{e_{i}}\left(m, t e_{j}\right)\right)=f\left(m, t e_{j}+e_{i}\right), \quad \text { a. e. } t \in(0,1),\{i, j\}=\{1,2\} .
$$

This holds true for instance if $f(m, \cdot)$ is in $W_{\sharp}^{1,2}(Y)$ with a trace independent of $m$.

Remark 4.8. The constancy property in iii) will be crucial in the homogenization process. It will ensure that the internal resonances associated to each rod can be handled separately. An abstract proof can be deduced directly from the ergodicity of the dynamical system (see [26]). Here in order to help for a more intuitive understanding, we proceed directly in our particular case.

Proof. Let $f \in H_{s}^{1}(\Omega)$. In view of the assertion iv) of Proposition 4.4, we know that for $\mathbb{P}$ almost all $(m, y)$, the function $x \mapsto \tilde{f}(m, x+y)\left(=f\left(T_{x}(m, y)\right)\right.$ belongs to $W_{\text {loc }}^{1,2}\left(\mathbb{R}^{2}\right)$. Since this property is invariant by translation, it follows that for $\Pi$ a.e. $m$, it holds $\tilde{f}(m, \cdot) \in W_{\text {loc }}^{1,2}\left(\mathbb{R}^{2}\right)$. Furthermore, by (4.11), we know that

$$
\nabla_{x} \tilde{f}(m, x+y)=\nabla^{s} f\left(T_{x}(m, y)\right) \quad \text { for a.a. }(x, y) \in \mathbb{R}^{2} \times Y .
$$

In particular, let us choose a Lebesgue point $x \in Y$ for both $\nabla_{x} \tilde{f}(m, \cdot)$ and $\nabla^{s} f(m, \cdot)$ and average the equality above on a small ball $\{|y| \leq r\}$ with $r<\operatorname{dist}(x, \partial Y)$ so that $T_{x}(m, y)=(m, x+y)$. Then by taking the limit as $r \rightarrow 0$ we obtain (4.16) holding for a.e. $x \in Y$. As a consequence, $f \in L^{2}\left(\Pi, \boldsymbol{\pi} ; W^{1,2}(Y)\right)$ with

$$
\int_{\Pi}\|f(m, \cdot)\|_{H^{1}(Y)}^{2} \mathrm{~d} \Pi=\int_{\Pi}\left(\int_{Y}|f(m, y)|^{2}+\left|\nabla^{s} f(m, y)\right|^{2} \mathrm{~d} y\right) \mathrm{d} \Pi=\|f\|_{H_{s}^{1}(\Omega)}^{2}<+\infty .
$$


Conversely, if for a.a. $m \in \Pi$, the function $\tilde{f}(m, \cdot)$ given in (4.15) belongs to $W_{\text {loc }}^{1,2}\left(\mathbb{R}^{2}\right)$, then it is also the case for the translated maps $x \rightarrow \tilde{f}(m, x+y)=f\left(T_{x}(m, y)\right)$ for every $y \in Y$. Moreover we have $\nabla_{x}\left(f\left(T_{x}(m, y)\right)=\right.$ $F\left(T_{x}(m, y)\right)$ where $F=\nabla_{x} \tilde{f}$ is an element of $\left(L^{2}(\Omega)\right)^{2}$. Indeed as $\tilde{f}(m, \cdot)$ agrees with $f(m, \cdot)$ on $Y$, one has

$$
\mathbb{E}\left(|F|^{2}\right)=\int_{\Pi}\left(\int_{Y}\left|\nabla_{x} f\right|^{2} \mathrm{~d} x\right) \mathrm{d} \pi \leq \int_{\Pi}\|f(m, \cdot)\|_{W^{1,2}(Y)}^{2}<+\infty .
$$

Therefore the random field $(\omega, x) \mapsto \nabla_{x} f\left(T_{x}(\omega)\right)$ is statistically homogeneous on $\Omega \times \mathbb{R}^{2}$ and we conclude that $f \in H_{s}^{1}(\Omega)$ by applying the assertion iv) or Proposition 4.4.

The assertion ii) is a direct consequence of Corollary 4.5. Let us prove iii). From the assertion i), we deduce that the function $f \in H_{s}^{1}(\Omega)$ is such that for $\pi$ almost all $m \in \Pi, f(m, \cdot)$ as an element of $W^{1,2}(Y)$ has a vanishing gradient on the connected set $Y \backslash B\left(\boldsymbol{\theta}_{0}(m), \boldsymbol{\rho}_{0}(m)\right)$. Therefore there exists a constant $c(m)$ such that $f(m, \cdot)=c(m)$ a.e. on $Y \backslash B\left(\boldsymbol{\theta}_{0}(m), \boldsymbol{\rho}_{0}(m)\right)$. In addition, in order to fit with the trace compatibility condition (4.18), we need that for a.e $t \in(0,1)$ and for $\{i, j\}=\{1,2\}$

$$
c(m)=f\left(m, e_{i}+t e_{j}\right)=f\left(T_{e_{i}}\left(m, t e_{j}\right)\right)=f\left(\left(m_{k+e_{i}}\right)_{k}, t e_{j}\right)=c\left(\left(m_{k+e_{i}}\right)_{k}\right) .
$$

From this follows that the function $m \mapsto c(m)$ is shift invariant over $(M, \pi)$, thus constant by ergodicity. This yields (4.17).

\section{TWO-SCALE ANALYSis OF THE DIFFRACTION PROBLEM}

In this section and all along the rest of the paper, we will consider a subset $\tilde{\Omega} \subset \Omega$ of full measure (that is $\mathbb{P}(\tilde{\Omega})=1$ ), the elements of which will be referred as "typical events".

For such $\tilde{\omega} \in \tilde{\Omega}$, we ask first that:

- property (4.2) holds for all $f \in C(\Omega)$ (according to the terminology in [26], $x \mapsto T_{x}(\tilde{\omega})$ is a "typical" trajectory of the dynamical system)

- for generic elements $f$ in a dense subset of $H_{s}^{1}(\Omega)$ the realizations $x \mapsto f\left(T_{x}(\tilde{\omega})\right)$ belong to $W_{\text {loc }}^{1,2}\left(\mathbb{R}^{2}\right)$ and satisfy the chain rule (4.11)

- for $\Psi$ in a dense subset of $D\left(\operatorname{div}^{\mathrm{s}}\right)$, it holds $\operatorname{div}_{\mathrm{x}}\left(\psi\left(T_{x}(\tilde{\omega})\right)=\operatorname{div}^{\mathrm{s}} \psi\left(T_{x} \tilde{\omega}\right)\right.$ in $L_{\mathrm{loc}}^{2}\left(\mathbb{R}^{2}\right)$.

We notice that the existence of such a set $\tilde{\Omega}$ of full measure is straightforward by exploiting the separability of $C(\Omega)$ and, in order to fit to two last items, by applying Proposition 4.4 (resp. Cor. 4.5) to a countable dense subset of elements $f$ in the Hilbert space $H_{s}^{1}\left(\right.$ resp. $\left.\psi \in D\left(\operatorname{div}^{\mathrm{s}}\right)\right)$.

\subsection{Two-scale convergence}

As our aim is to obtain convergence results almost surely, we will use a recent variant of the notion of "stochastic two-scale convergence in the mean" introduced for the first time in 1994 in [7]. Precisely we will use the notion of "realizationwise two-scale convergence" as introduced in [26] in the context of the homogenization of random thin structures and singular measures. The definition below is given using a reference open ball $B_{R}$ of $\mathbb{R}^{2}$ (where in practice the radius $R$ is so large that $B_{R} \supset \mathcal{B}$ ).

Definition 5.1 (stochastic two-scale convergence). We say that a sequence $f_{\eta} \in L^{2}\left(B_{R}\right)$ two-scale converges to $f_{0} \in L^{2}\left(B_{R} \times \Omega, \mathcal{L}^{2} \otimes \mathbb{P}\right)$, and we write $f_{\eta}(x) \longrightarrow f_{0}(x, \omega)$ if for all $\tilde{\omega} \in \tilde{\Omega}$ it holds

$$
\lim _{\eta \rightarrow 0} \int_{B_{R}} f_{\eta}(x) \varphi(x) \psi\left(T_{\frac{x}{\eta}} \tilde{\omega}\right) \mathrm{d} x=\mathbb{E}\left(\int_{B_{R}} f_{0}(x, \cdot) \varphi(x) \psi(\cdot) \mathrm{d} x\right)
$$


for any $\varphi \in C_{c}^{\infty}\left(B_{R}\right)$ and $\psi \in L^{2}(\Omega)$. The sequence will be said to be strongly two-scale convergent (denoted $f_{\eta} \rightarrow f_{0}$ ) if in addition

$$
\limsup _{\eta \rightarrow 0} \int_{B_{R}}\left|f_{\eta}(x)\right|^{2} \mathrm{~d} x \leq \int_{B_{R} \times \Omega}\left|f_{0}(x, \omega)\right|^{2} \mathrm{~d} x \mathbb{P}(\mathrm{d} \omega) .
$$

A justification of definition (5.1) is that any sequence $f_{\eta}$ has a two-scale converging subsequence provided it is bounded in $L^{2}\left(B_{R}\right)$. Notice that the existence of a two-scale limit implies that the left hand member of (5.1) is independent of the choice of $\tilde{\omega} \in \tilde{\Omega}$. In that case, in order to identify such a two-scale limit, it is enough to restrict to a particular event $\tilde{\omega} \in \tilde{\Omega}$.

The main feature of strong two-scale convergence is that we have the following product rule:

$$
f_{\eta} \rightarrow f_{0}, g_{\eta} \rightarrow g_{0} \Longrightarrow f_{\eta} g_{\eta} \rightarrow \mathbb{E}\left(f_{0} g_{0}\right) \quad \text { in the distributional sense on } B_{R} \text {. }
$$

This applies in particular for $f_{\eta}$ of the kind $f_{\eta}(x)=\varphi(x) \psi\left(T_{\frac{x}{\eta}} \tilde{\omega}\right)$ with $\tilde{\omega}$ arbitrary in $\tilde{\Omega}, \varphi \in C\left(B_{R}\right)$ and $\psi \in L^{2}(\Omega)$ (in that case, there holds $f_{\eta} \rightarrow \varphi(x) \psi(\omega)$ ). Furthermore the strong convergence $f_{\eta} \rightarrow f_{0}$ implies that

$$
\lim _{\eta \rightarrow 0^{+}} \int_{B_{R}}\left|f_{\eta}(x)-f_{0}\left(x, T_{\frac{x}{\eta}} \tilde{\omega}\right)\right|^{2} \mathrm{~d} x=0
$$

whenever $f_{0}$ is suitably regular.

Remark 5.2. If in definition (4.1) we choose $\mathbf{p}$ to be a Dirac mass (determinist case), we fall on the classical notion of two-scale (resp. strong two-scale) convergence which has been introduced years ago in $[1,19]$.

Remark 5.3. Let us emphasize that the notion of stochastic two-scale convergence introduced above will be applied to to each particular realization $f_{\eta}(x, \tilde{\omega})$ of a sequence of random functions we wish to study, providing then a two-scale limit $f_{0}(x, \omega, \tilde{\omega})$ depending a priori of the realization $\tilde{\omega}$ and of the associated extracted subsequence. This apparent drawback disappears in the context of effective media theory when the homogenization procedure provides a unique two-scale limit $f_{0}(x, \omega)$. Note that this uniqueness argument is used in the context of periodic homogenization as well.

\subsection{Differential characterization of the two-scale limits}

In this subsection, we fix an event $\tilde{\omega} \in \tilde{\Omega}$ and to simplify notions we denote:

$$
u_{\eta}(x)=u_{\eta}(x, \tilde{\omega}), \quad a_{\eta}(x)=a_{\eta}(x, \tilde{\omega}), \quad D_{\eta}=D_{\eta}(\tilde{\omega}) .
$$

We assume a priori that the sequences $\left(u_{\eta}\right),\left(\eta \nabla u_{\eta}\right)$ and $\left(1_{B_{R} \backslash \mathcal{D}_{\eta}} \nabla u_{\eta}\right)$ are bounded in $L^{2}\left(B_{R}\right)$ and satisfy

$$
u_{\eta} \longrightarrow u_{0}(x, \omega), \quad \eta \nabla u_{\eta} \longrightarrow P_{0}(x, \omega), \quad 1_{B_{R} \backslash \mathcal{D}_{\eta}} \nabla u_{\eta} \longrightarrow \chi_{0}(x, \omega),
$$

for suitable functions $u_{0} \in L^{2}\left(B_{R} \times \Omega, \mathcal{L}^{2} \otimes \mathbb{P}\right)$ and $\chi_{0}, P_{0} \in\left(L^{2}\left(B_{R} \times \Omega, \mathcal{L}^{2} \otimes \mathbb{P}\right)\right)^{2}$. Such an assumption will be justified later on in Section 6 where the $L^{2}$ bounds are established as well as the uniqueness of the two-scale limits (which do not depend on the choice of $\tilde{\omega}$ ).

Proposition 5.4. Let $u_{0}, P_{0}$ and $\chi_{0}$ be the two-scale limit defined in (5.4). Then for $\mathrm{d} x \otimes \mathbb{P}$-almost every $(x, \omega) \in B_{R} \times \Omega$ we have the following relations:

$$
\begin{aligned}
P_{0}(x, \omega)=0 & \text { in }\left(\mathcal{B} \times \Sigma^{*}\right) \cup\left[\left(B_{R} \backslash \mathcal{B}\right) \times \Omega\right], \\
\nabla^{s} u_{0}(x, \omega)=P_{0}(x, \omega) & \text { in } B_{R} \times \Omega, \\
\chi_{0}(x, \omega)=0 & \text { in } \mathcal{B} \times \Sigma, \\
\operatorname{div}^{\mathrm{s}} \chi_{0}(x, \omega)=0 & \text { in } B_{R} \times \Omega .
\end{aligned}
$$


Proof. For $\varphi \in C_{c}^{\infty}\left(B_{R}\right)$ and $\psi$ in a dense subset of $D\left(\operatorname{div}^{\mathrm{s}}\right)$, we use $\varphi(x) \psi\left(T_{\frac{x}{\eta}} \tilde{\omega}\right)$ as test function in order to express the two-scale convergence $\eta \nabla u_{\eta} \longrightarrow P_{0}$. We obtain:

$$
\int_{B_{R} \times \Omega} P_{0}(x, \omega) \varphi(x) \psi(\omega) \mathrm{d} x \mathrm{~d} \mathbb{P}(\omega)=\lim _{\eta \rightarrow 0} \eta \int_{B_{R}} \nabla u_{\eta} \cdot \varphi(x) \psi\left(T_{\frac{x}{\eta}} \tilde{\omega}\right) \mathrm{d} x .
$$

Let assume that $(\varphi, \psi)$ is supported in $\mathcal{B} \times \Sigma^{*}$. It follows from (4.3) that the integration domain in the right hand side of (5.9) reduces to $\mathcal{B} \backslash \mathcal{D}_{\eta}$. The convergence $1_{B_{R} \backslash \mathcal{D}_{\eta}} \nabla u_{\eta} \longrightarrow \chi_{0}$ implies that the right hand side of (5.9) vanishes as $\eta \rightarrow 0$. First part of claim (5.5) follows by a trivial localization argument. The second part is deduce by the same kind of argument after notice that $\nabla u_{\eta}$ remains bounded in $L^{2}\left(B_{R} \backslash \mathcal{B}\right)$.

In order to prove (5.6), we integrate by parts the right hand member of (5.9). We obtain

$$
\eta \int_{B_{R}} \nabla u_{\eta} \cdot \varphi(x) \psi\left(T_{\frac{x}{\eta}} \tilde{\omega}\right) \mathrm{d} x=-\int_{B_{R}} u_{\eta}\left(\eta \nabla \varphi(x) \cdot \psi\left(T_{\frac{x}{\eta}} \tilde{\omega}\right)+\varphi(x)\left(\operatorname{div}{ }_{s} \psi\right)\left(T_{\frac{x}{\eta}} \tilde{\omega}\right)\right) \mathrm{d} x
$$

where the stochastic divergence appears by applying the chain rule formula (4.11). Passing to the limit $\eta \rightarrow 0$, by using (5.9) and the convergence $u_{\eta} \longrightarrow u_{0}$, we deduce that

$$
\int_{B_{R} \times \Omega} P_{0}(x, \omega) \varphi(x) \psi(\omega) \mathrm{d} x \mathrm{~d} \mathbb{P}(\omega)=-\int_{B_{R} \times \Omega} u_{0}(x, \omega) \varphi(x) \operatorname{div}{ }_{s} \psi(\omega) \mathrm{d} x \mathrm{~d} \mathbb{P}(\omega) .
$$

The claim (5.6) follows by exploiting the integration by parts formula (4.9).

Recalling (4.4), claim (5.7) is a consequence of the two-scale convergence $1_{B_{R} \backslash \mathcal{D}_{\eta}} \nabla u_{\eta} \longrightarrow \chi_{0}(x, \omega)$ evaluated with test functions of the kind $\varphi(x) \psi\left(T_{\frac{x}{\eta}} \tilde{\omega}\right)$ being $\psi$ supported in $\Sigma$ and $\varphi$ compactly supported in $\mathcal{B}$ (that is in $\mathcal{B}_{\eta}(\tilde{\omega})$ for all $\eta$ small enough).

Next we multiply (1.4) by function $\eta \varphi(x) \psi\left(T_{\frac{x}{\eta}} \tilde{\omega}\right)$ with $(\varphi, \psi) \in C_{c}^{\infty}\left(B_{R}\right) \times H_{s}^{1}(\Omega)$, and integrate by parts with the help of chain rule formula (4.11). It comes

$$
\int_{B_{R}} a_{\eta} \nabla u_{\eta} \cdot\left(\varphi(x) \nabla^{\mathrm{s}} \psi\left(T_{\frac{x}{\eta}} \tilde{\omega}\right)+\eta \psi\left(T_{\frac{x}{\eta}} \tilde{\omega}\right) \nabla \varphi\right)=\eta k_{0}^{2} \int_{B_{R}} u_{\eta} \varphi(x) \psi\left(T_{\frac{x}{\eta}} \tilde{\omega}\right) .
$$

Then noticing that $a_{\eta}$ is of order $\eta^{2}$ in $\mathcal{D}_{\eta}$, we infer from (5.4) that

$$
\int_{B_{R} \times \Omega} \chi_{0}(x, \omega) \cdot \varphi(x) \nabla^{\mathrm{s}} \psi(\omega) \mathrm{d} x \mathrm{~d} \mathbb{P}(\omega)=0 .
$$

Relation (5.8) follows by localizing in $x$ and by using once more (4.9).

\subsection{Identification of $\mathrm{u}_{0}(\mathrm{x}, \cdot)$}

Proposition 5.5. Let $u_{0}$ be the two-scale limit in (5.4). Then $u_{0} \in L^{2}\left(B_{R} ; H_{s}^{1}(\Omega)\right)$ and there exists a function $u=u(x)$ in $L^{2}\left(B_{R}\right)$ (independent of $\omega$ ) such that:

i)

ii)

$$
\begin{gathered}
u_{0}(x, \omega)=u(x) \quad \text { a.e. in }\left(\mathcal{B} \times \Sigma^{*}\right) \cup\left[\left(B_{R} \backslash \mathcal{B}\right) \times \Omega\right] \\
\operatorname{div}^{\mathrm{s}}\left(\varepsilon_{0}^{-1}(\omega) \nabla^{\mathrm{s}} u_{0}(x, \omega)\right)+k_{0}^{2} u_{0}(x, \omega)=0 \quad \text { in } \quad \mathcal{B} \times \Sigma .
\end{gathered}
$$

iii) Assume furthermore that condition (2.10) holds and let $\Lambda$ be defined by (2.7). Then

$$
u_{0}(x, \omega)=u(x) \Lambda(\omega) \quad \text { a.e. in } \mathcal{B} \times \Omega .
$$


Proof. From equation (5.6) we deduce that $\nabla^{s} u_{0}$ is an element of $L^{2}\left(B_{R} \times \Omega\right)$ and therefore $u_{0} \in L^{2}\left(B_{R} ; H_{s}^{1}(\Omega)\right)$. In addition, by (5.5), we have $\nabla^{s} u_{0}(x, \cdot)=0$ in $\left(\mathcal{B} \times \Sigma^{*}\right) \cup\left(\left(B_{R} \backslash \mathcal{B}\right) \times \Omega\right)$. Then claim i) is a consequence of the assertion iii) of Proposition 4.6. Next in order to prove claim ii), we consider $\varphi \in C_{c}^{\infty}(\mathcal{B})$ and $\psi \in C^{0}(\Omega)$ running in a dense subset of the subspace $\left\{\psi \in H_{s}^{1}(\Omega): \psi=0\right.$ in $\left.\Sigma^{*}\right\}$. Then we multiply equation (1.4) (for $\omega=\tilde{\omega})$ by test function $\varphi(x) \psi\left(T_{\frac{x}{\eta}} \tilde{\omega}\right)$ and integrate over $\mathcal{B}$. Then taking into account chain rule (4.11), we are led to

$$
\int_{\mathcal{B}} a_{\eta}(x, \tilde{\omega}) \nabla u_{\eta} \cdot\left(\nabla \varphi(x) \psi\left(T_{\frac{x}{\eta}} \tilde{\omega}\right)+\frac{1}{\eta} \varphi(x) \nabla^{s} \psi\left(T_{\frac{x}{\eta}} \tilde{\omega}\right)\right) \mathrm{d} x=k_{0}^{2} \int_{\mathcal{B}} u_{\eta} \varphi(x) \psi\left(T_{\frac{x}{\eta}} \tilde{\omega}\right) \mathrm{d} x \mathrm{~d} \mathbb{P} .
$$

In view of (4.5) and since $\psi$ vanishes outside $\Sigma$ and $\varphi$ vanish in $\mathcal{B}_{\eta}(\tilde{\omega})$ for $\eta$ small enough, we may substitute $a_{\eta}(x, \tilde{\omega})$ with $\eta^{2}\left(\varepsilon_{0}\left(T_{\frac{x}{\eta}} \tilde{\omega}\right)\right)^{-1}$ in the equality above. Then we may pass to the limit $\eta \rightarrow 0$ by using the weak two-scale convergences in (5.4) and relation (5.6):

$$
\int_{\mathcal{B} \times \Omega} \varepsilon_{0}^{-1}(\omega) P_{0}(x, \omega) \cdot \nabla^{\mathrm{s}} \psi(\omega) \varphi(x) \mathrm{d} x \mathrm{~d} \mathbb{P}=k_{0}^{2} \int_{\mathcal{B} \times \Omega} u_{0}(x, \omega) \varphi(x) \psi(\omega) \mathrm{d} x \mathrm{~d} \mathbb{P} .
$$

Eventually, since $P_{0}=\nabla^{s} u_{0}$ (by (5.6)) and by the arbitrariness of function $\varphi$, we are led to relation

$$
\int_{\Omega} \varepsilon_{0}^{-1}(\omega) \nabla^{\mathrm{s}} u_{0}(x, \omega) \cdot \nabla^{\mathrm{s}} \psi(\omega) \mathrm{d} \mathbb{P}=k_{0}^{2} \int_{\Omega} u_{0}(x, \omega) \psi(\omega) \mathrm{d} \mathbb{P},
$$

holding for a.a. $x \in \mathcal{B}$ and for all $\Psi \in H_{s}^{1}(\Omega)$ vanishing outside $\Sigma$. In view of the characterization (4.9), this is precisely equivalent to relation of assertion ii).

Let us prove now the assertion iii). By using the claim i) of Proposition 4.6 (namely relation (4.16)) and recalling that $\Sigma=\left\{(y, m): y \in B\left(\boldsymbol{\theta}_{0}(m), \boldsymbol{\rho}_{0}(m)\right)\right\}$, we may rewrite equation (5.11) as a boundary value problem in $B\left(\boldsymbol{\theta}_{0}(m), \boldsymbol{\rho}_{0}(m)\right)$. For a.e. $(x, m) \in \mathcal{B} \times \Pi$, the function $\varphi:=u_{0}(x, m, \cdot)$ belongs to $W^{1,2}(Y)$, agrees with constant $u(x)$ in $B\left(\boldsymbol{\theta}_{0}(m), \boldsymbol{\rho}_{0}(m)\right)$ and solves

$$
\begin{cases}\Delta_{y} \varphi+k_{0}^{2} \varepsilon_{0}(m) \varphi=0 & \text { in } B\left(\boldsymbol{\theta}_{0}(m), \boldsymbol{\rho}_{0}(m)\right), \\ \varphi=u(x) & \text { on } \partial B\left(\boldsymbol{\theta}_{0}(m), \boldsymbol{\rho}_{0}(m)\right) .\end{cases}
$$

This is precisely the Helmholtz resonator problem depicted in the Appendix. By applying Lemma A.1 with $\rho:=\boldsymbol{\rho}_{0}(m), \alpha:=k_{0}^{2} \varepsilon_{0}(m)$, we find that the unique solution reads $\varphi=u(x) w\left(y-\boldsymbol{\theta}_{0}(m)\right)$ where $w$ is given by the series (A.1). The assumption (2.10) ensures that $\alpha \rho^{2}$ does not belong to $\boldsymbol{\sigma}_{0}$ (spectrum of Laplace operator on the unit disk). Eventually, owing to $(2.7)$, one has $\Lambda(m, \cdot)=w\left(\cdot-\boldsymbol{\theta}_{0}(m)\right)$ for $\pi$ a.e. $m \in \Pi$ and we are led to relation (5.10).

Remark 5.6. As a consequence of Propositions 5.5 we know that $u_{0}(x, \cdot) \in H_{s}^{1}(\Omega)$ for a.e. $x \in \mathcal{B}$. Thus relation (5.10) implies $\Lambda \in H_{s}^{1}(\Omega)$ unless $u \equiv 0$. To conclude this, two hypothesis are needed:

- The condition (2.10), saying that $\mathbf{p}(\{(\theta, \rho, \varepsilon) \in M, \Im(\varepsilon)>0\})>0$;

- The two-scale convergence (5.4); which will be in fact equivalent, up to a subsequence, to the $L^{2}$ bound (6.1) (see Lem. 6.2).

Accordingly, the property $\Lambda \in H_{s}^{1}(\Omega)$ appears to be a necessary condition in order to have (6.1). Actually, we will have to assume conditions (2.9), (2.10) in order to ensure that (6.1) holds for $\mathbb{P}$-almost all $\tilde{\omega} \in \tilde{\Omega}$ (see Prop. 6.9). As can be seen in Lemma 5.7 below, the fact that $\Lambda$ belongs to $H_{s}^{1}(\Omega)$ can be derived directly from the assumption (2.9) (in fact we need it merely for $r=0$ ).

Lemma 5.7. Under condition (2.9), we have $\Lambda \in H_{s}^{1}(Y)$ and it holds for every $\tilde{\omega} \in \tilde{\Omega}$ :

$$
\begin{gathered}
\lim _{\eta \rightarrow 0^{+}} \int_{\mathcal{B}}\left|\Lambda\left(T_{\frac{x}{\eta}} \tilde{\omega}\right)\right|^{2} \mathrm{~d} x=|\mathcal{B}| \int_{\Omega}|\Lambda(\omega)|^{2} \mathbb{P}(\mathrm{d} \omega), \\
\lim _{\eta \rightarrow 0^{+}} \int_{\mathcal{B}}\left|\nabla^{\mathrm{s}} \Lambda\left(T_{\frac{x}{\eta}} \tilde{\omega}\right)\right|^{2} \mathrm{~d} x=|\mathcal{B}| \int_{\Omega}\left|\nabla^{\mathrm{s}} \Lambda(\omega)\right|^{2} \mathbb{P}(\mathrm{d} \omega) .
\end{gathered}
$$


Proof. We notice that $\Lambda(m, \cdot)=1$ near $\partial Y$ and by Remark 4.7, in order to show that $\Lambda \in H_{s}^{1}(\Omega)$, we have only to check that $\Lambda \in L^{2}\left(\Pi, \pi ; W^{1,2}(Y)\right)$. Recalling that $\int_{Y} \varphi_{n} \varphi_{m} \mathrm{~d} y=\delta_{n m}$ and $\int_{Y}\left|\nabla \varphi_{n}\right|^{2} \mathrm{~d} y=\lambda_{n}$, we can compute

$$
\|\Lambda-1\|_{L^{2}(\Omega)}^{2}=\sum_{n \in I_{0}} \alpha_{n} c_{n}^{2}, \quad\left\|\nabla_{y} \Lambda\right\|_{L^{2}(\Omega)}^{2}=\sum_{n \in I_{0}} \beta_{n} \lambda_{n} c_{n}^{2}
$$

where we denote

$$
c_{n}:=\int_{B_{1}} \varphi_{n} \mathrm{~d} y, \quad \alpha_{n}:=\int_{M}\left(\frac{k_{0}^{2} \varepsilon \rho^{3}}{\lambda_{n}-k_{0}^{2} \varepsilon \rho^{2}}\right)^{2} \mathrm{~d} \mathbf{p}, \quad \beta_{n}:=\int_{M}\left(\frac{k_{0}^{2} \varepsilon \rho^{2}}{\lambda_{n}-k_{0}^{2} \varepsilon \rho^{2}}\right)^{2} \mathrm{~d} \mathbf{p} .
$$

From condition (2.9) (which clearly holds also if $r=0$ ) and the fact that $\mathbf{p}$ is compactly supported, we infer that $\sup _{n} \alpha_{n}<+\infty$. Furthermore, since $\lambda_{n} \rightarrow \infty$ as $n \rightarrow \infty$, there exists a constant $C>0$ such that for $n$ sufficiently large

$$
\lambda_{n}^{2} \beta_{n}=\int_{M}\left(\frac{k_{0}^{2} \varepsilon \rho^{2}}{1-\frac{k_{0}^{2} \varepsilon \rho^{2}}{\lambda_{n}}}\right)^{2} \leq C .
$$

Thus, as $\sum_{n}\left|c_{n}\right|^{2}<+\infty$ and in view of equalities (5.13), we deduce that $\Lambda \in H_{s}^{1}(\Omega)$ with $\nabla^{s} \Lambda(m, \cdot)=\nabla_{y} \Lambda(m, \cdot)$ (see $(4.16))$.

Let us prove now (5.12). By applying Lemma 4.1 and the chain rule (4.11), we find that the maps $x \mapsto \Lambda\left(T \frac{x}{\eta} \tilde{\omega}\right)$ and $x \mapsto \nabla^{s} \Lambda\left(T_{\frac{x}{\eta}} \tilde{\omega}\right)$ are in $L^{2}(\mathcal{B})$ and $\left(L^{2}(\mathcal{B})\right)^{2}$ respectively. Accordingly, by (4.2), we infer that (5.12) holds for every $\omega \in \tilde{\Omega}$.

\subsection{Identification of vector field $\chi_{0}$}

Let us point out that $\chi_{0}(x, \omega)$ defined by $(5.4)$ is associated up to a $\pi / 2$ rotation to the (transverse) electric field which is oscillating at scale $\eta$ in the obstacle $\mathcal{B}$. In the following we will use the average of $\chi_{0}(x, \cdot)$ that is

$$
j(x)=\left(j_{1}, j_{2}\right)=\mathbb{E}\left(\chi_{0}\right) .
$$

We are going to determine explicitly $\chi_{0}$ and $j$ in term of the effective permittivity tensor $\varepsilon^{\text {eff }}$ given in (2.3) and of the local gradient $\nabla u(x)$ of function $u$ defined in Proposition 5.5 (which, roughly speaking, describes the slowly varying magnetic field "outside" the rods).

Proposition 5.8. Let us assume that (2.10) and (5.4) hold. Let $\mathbf{A}$ be the tensor defined in (2.12) and let u, $\chi_{0}, j$ be the functions introduced respectively in Proposition 5.5, (5.4) and (5.14). Then

i) We have the relation:

$$
\chi_{0}(x, \omega)= \begin{cases}j(x) & \text { in }\left(B_{R} \backslash \mathcal{B}\right) \times \Omega \\ j_{1}(x) \sigma^{1}(\omega)+j_{2}(x) \sigma^{2}(\omega) & \text { in } \mathcal{B} \times \Omega\end{cases}
$$

where for $i \in\{1,2\}, \sigma^{i} \in L_{\text {sol }}^{2}(\Omega)$ is the unique solution of $(2.3)$ with $\mathbb{E}(\sigma)=e_{i}$.

ii) $u$ belongs to $H^{1}\left(B_{R}\right)$ and the following constitutive relation holds

$$
j(x)=\mathbf{A}(\mathbf{x}) \nabla \mathbf{u}= \begin{cases}\nabla u(x) & \text { if } x \in B_{R} \backslash \mathcal{B} \\ \left(\varepsilon^{\mathrm{eff}}\right)^{-1} \nabla u(x) & \text { if } x \in \mathcal{B}\end{cases}
$$


Proof. Let $\varphi \in C_{c}^{\infty}\left(B_{R}\right)$ and $\boldsymbol{\psi}$ in a dense subset of $L_{\text {sol }}^{2}(\Omega)$ such that $\psi=0$ on $\Sigma$. By (4.3), the function $x \mapsto \boldsymbol{\psi}\left(T_{\frac{x}{\eta}} \tilde{\omega}\right)$ vanishes in $\mathcal{D}_{\eta}(\tilde{\omega})$ whereas, by Corollary 4.5 , the vector field $x \mapsto \psi\left(T_{\frac{x}{\eta}} \tilde{\omega}\right)$ is divergence free. Thus:

$$
\begin{aligned}
& \int_{B_{R}}\left[\nabla_{x} u_{\eta}(x, \tilde{\omega}) 1_{B_{R} \backslash \mathcal{D}_{\eta}(\tilde{\omega})}(x)\right] \cdot \varphi(x) \boldsymbol{\psi}\left(T_{\left.\frac{x}{\eta} \tilde{\omega}\right) \mathrm{d} x}\right. \\
&=\int_{B_{R}} \nabla_{x} u_{\eta}(x, \tilde{\omega}) \cdot \varphi(x) \psi\left(T_{\frac{x}{\eta}} \tilde{\omega}\right) \mathrm{d} x=-\int_{B_{R}} u_{\eta}(x, \tilde{\omega})\left[\nabla \varphi(x) \cdot \boldsymbol{\psi}\left(T_{\frac{x}{\eta}} \tilde{\omega}\right)\right] \mathrm{d} x .
\end{aligned}
$$

Let us pass to the limit $\eta \rightarrow 0$. In view of the two-scale convergences in $(5.4)$ and noticing that $u_{0}(x, \cdot) \psi=$ $u(x) \psi($ see assertion i) of Prop. 5.5), we obtain

$$
\begin{aligned}
\int_{B_{R} \times \Omega} \chi_{0}(x, \omega) \cdot \varphi(x) \boldsymbol{\psi}(\omega) \mathrm{d} x \mathrm{~d} \mathbb{P} & =-\int_{B_{R} \times \Omega} u_{0}(x, \omega) \nabla_{x} \varphi(x) \cdot \boldsymbol{\psi}(\omega) \mathrm{d} x \mathrm{~d} \mathbb{P} \\
& =-\int_{B_{R}} u(x) \nabla_{x} \varphi(x) \mathrm{d} x \cdot \int_{\Omega} \boldsymbol{\psi}(\omega) \mathrm{d} \mathbb{P} .
\end{aligned}
$$

Choosing first $\varphi$ to be compactly supported in $\mathcal{B}$ and by localization, we deduce from (5.17) that for a.e. $x \in \mathcal{B}$, the vector field $\chi_{0}(x, \cdot)$ satisfies the equation

$$
\mathbb{E}\left(\chi_{0}(x, \cdot) \cdot \boldsymbol{\psi}\right)=0 \quad \text { for all } \boldsymbol{\psi} \in L_{\text {sol }}^{2}(\Omega) \text { such that } \boldsymbol{\psi}=0 \text { in } \Sigma \text { and } \mathbb{E}(\boldsymbol{\psi})=0 .
$$

Recalling that $\chi_{0}(x, \cdot)$ vanishes on $\Sigma$ (see $\left.(5.7)\right)$ and in view of $(5.14)$, we find that $\chi_{0}(x, \cdot)$ satisfies the variational characterization of the solution of problem (2.3) for $z=j(x)$. Accordingly $\chi_{0}(x, \cdot)$ agrees for $x \in \mathcal{B}$ with the linear decomposition appearing in (5.15).

In order to prove that $\chi_{0}(x, \cdot)=j(x)$ for $x \in B_{R} \backslash \mathcal{B}$, we take now $\varphi$ to be compactly supported in $B_{R} \backslash \overline{\mathcal{B}}$ and observe that relation (5.17) holds true for all $\boldsymbol{\psi} \in L_{\mathrm{sol}}^{2}(\Omega)$ (it is not necessary that $\boldsymbol{\psi}$ vanishes in $\Sigma$ ). By localization, we find that, for a.e. $x \in B_{R} \backslash \mathcal{B}$, it holds $\mathbb{E}\left(\chi_{0}(x, \cdot) \cdot \psi\right)=0$ for $\boldsymbol{\psi} \in L_{\text {sol }}^{2}(\Omega)$ with vanishing average. As constant vector fields belong to $L_{\mathrm{sol}}^{2}(\Omega)$, we may choose in particular $\psi_{0}=\chi_{0}(x, \cdot)-j(x)$ so that

$$
\mathbb{E}\left(\left|\chi_{0}(x, \cdot)-j(x)\right|^{2}\right)=\mathbb{E}\left(\chi_{0}(x, \cdot) \cdot \boldsymbol{\psi}_{0}\right)-j(x) \cdot \mathbb{E}\left(\boldsymbol{\psi}_{0}\right)=0 .
$$

This ends the proof of assertion i). Let us prove now the assertion ii). To that aim, let us apply (5.17) with $\boldsymbol{\psi}=\sigma^{i}$ for $i=1,2$. Recalling that $\mathbb{E}\left(\sigma^{i}\right)=e_{i}$, we get

$$
\int_{B_{R} \times \Omega} \chi_{0}(x, \omega) \cdot \varphi(x) \sigma^{i}(\omega) \mathrm{d} x \mathrm{~d} \mathbb{P}=-\int_{B_{R}} u(x) \frac{\partial \varphi}{\partial x_{i}} \mathrm{~d} x
$$

holding for every $\varphi \in C_{c}^{\infty}\left(B_{R}\right)$. This allows to identify the distributional derivative of $u$ on $B_{R}$ as

$$
\frac{\partial u}{\partial x_{i}}=\mathbb{E}\left(\chi_{0}(x, \cdot) \cdot \sigma^{i}\right), i \in\{1,2\} .
$$

Clearly, by Cauchy-Schwartz inequality, the right hand member of $(5.18)$ is a function in $L^{2}\left(B_{R}\right)$. Moreover, by applying (5.15), we obtain that $\nabla u(x)=j(x)$ for a.e $x \in B_{R} \backslash \mathcal{B}$ whereas

$$
\frac{\partial u}{\partial x_{i}}=\mathbb{E}\left(\sigma^{1} \cdot \sigma^{i}\right) j_{1}+\mathbb{E}\left(\sigma^{2} \cdot \sigma^{i}\right) j_{2} \quad \text { a.e. in } \mathcal{B} .
$$

Then by the characterization (2.4) of tensor $\varepsilon^{\text {eff }}$, we conclude that relation (5.16) holds. 


\section{Proof of the MAin homogenization RESUlt}

This section is devoted to the proof of Theorem 2.3. Recall that $u_{\eta}(\cdot, \tilde{\omega})$ denotes the unique solution to $(1.4)$ and (1.6). It is associated with a particular realization $\tilde{\omega}$ of the random structure. From now on, we fix a reference ball $B_{R}$ of radius $R$ such that $\mathcal{B} \subset \subset B_{R}$ is compactly embedded and we will focus our attention to events $\tilde{\omega} \in \tilde{\Omega}$ such that:

$$
\sup _{\eta>0} \int_{B_{R}}\left|u_{\eta}(x, \tilde{\omega})\right|^{2} \mathrm{~d} x<\infty .
$$

As it will be shown later, the set of such events has in fact full measure in $\Omega$ (see Prop. 6.9). The proof of Theorem 2.3 is organized in three steps as follows:

Step 1. (convergence far from the obstacle). First we show that if, for a given $\tilde{\omega} \in \Omega$, we assume the bound (6.1), then possibly passing to a subsequence, $u_{\eta}(\cdot, \tilde{\omega})$ does converge uniformly as well as all its derivatives on every compact subsets of $\mathbb{R}^{2} \backslash \mathcal{B}$. In addition the radiation condition at infinity is preserved (see Prop. 6.1).

Step 2. (derivation of the homogenized problem). Still assuming (6.1) for a given $\tilde{\omega} \in \tilde{\Omega}$, we consider a weakly convergent subsequence $u_{\eta^{\prime}}(\cdot, \tilde{\omega}) \rightarrow u$ in $L^{2}\left(B_{R}\right)$. Then by using the two-scale analysis performed in Section 5 on $B_{R}$ and by applying to $u_{\eta^{\prime}}$ Step 1 on $\mathbb{R}^{2} \backslash \mathcal{B}$, we may pass to the limit in (1.4) obtaining that $u$ satifies the homogenized problem (2.11) (see Prop. 6.4). Under the condition (2.10) on the probability p, the solution to this homogenized problem turns out to be unique and independent of the realization $\tilde{\omega}$. As a consequence, the whole sequence $u_{\eta}(\cdot, \tilde{\omega})$ does two-scale converge to $u=u(x)$. At this point we have proved that all the conclusions of Theorem 2.3 (except the convergences in (2.15)) hold true for all $\tilde{\omega}$ satisfying (6.1).

Step 3. (strong two-scale convergence and $L^{2}$-bound). In this last step, we improve the convergence obtained in Step 2 under the additional assumption that $\mathbf{p}$ satisfies (2.9). More precisely we establish (see Prop. 6.6) that the sequence $u_{\eta}(\cdot, \tilde{\omega})$ actually converges strongly two-scale so that $(2.15)$ holds. So the proof of Theorem 2.3 is complete once the bound $(6.1)$ is established for $\mathbb{P}$-almost all $\tilde{\omega} \in \tilde{\Omega}$. This is done a posteriori (see Prop. 6.9) by using a contradiction argument in which we apply Proposition 6.6 to a renormalized sequence of solutions. Summarizing we are going to obtain the full homogenization result under the sole assumptions (2.9) and (2.10) on the probability distribution $\mathbf{p}$ of the dielectric rods.

\subsection{Behavior far of the obstacle}

This first step is related to the convergence of $u_{\eta}(\cdot, \tilde{\omega})$ at a positive distance from $\mathcal{B}$. We use the following result whose proof relies on the hypo-ellipticity of operator $\Delta+k_{0}^{2}$ and is very similar to that used in [4], Lemma 2.1 in the context of a 3D-diffraction problem.

Proposition 6.1. Fix $\tilde{\omega} \in \Omega$ and let $u_{\eta}:=u_{\eta}(\cdot, \tilde{\omega})$ be solving (1.4) with outgoing Sommerfeld radiation condition (1.6) for an incident wave $u^{\text {inc }}$. Let us assume that $u_{\eta} \rightarrow u=u(\cdot, \tilde{\omega})$ weakly in $L^{2}\left(B_{R}\right)$. Then the convergence $u_{\eta} \rightarrow u$ holds in $C^{\infty}(K)$ for any compact set $K \subset \mathbb{R}^{2} \backslash \overline{\mathcal{B}}$. Furthermore $u$ satisfies the Helmholtz equation $\Delta u+k_{0}^{2} u=0$ in $\mathbb{R}^{2} \backslash \overline{\mathcal{B}}$ and $\left(u-u^{\mathrm{inc}}\right)$ still satisfies (1.6). The same conclusions hold if in the radiation condition satisfied by $u_{\eta}$, we substitute the incident wave $u^{\text {inc }}$ with a sequence $\left(u_{\eta}^{\text {inc }}\right)$ such that $u_{\eta}^{\text {inc }} \rightarrow u^{\text {inc }}$ uniformly.

We can now derive an important additional energy estimate from which we can infer the two-scale convergences (5.4) for suitable subsequences and for some functions $u_{0}, P_{0}$ and $\chi_{0}$.

Lemma 6.2. Let $\tilde{\omega} \in \tilde{\Omega}$ such that condition (6.1) holds. Then it holds

$$
\sup _{\eta} \int_{B_{R}} a_{\eta}(x, \tilde{\omega})\left|\nabla_{x} u_{\eta}(x, \tilde{\omega})\right|^{2} \mathrm{~d} x<+\infty \text {. }
$$

As a consequence the sequences $\left(\eta \nabla u_{\eta}(x, \tilde{\omega})\right)$ and $\left(1_{B_{R} \backslash \mathcal{D}_{\eta}(\tilde{\omega})}(x) \nabla u_{\eta}(x, \tilde{\omega})\right)$ are bounded in $L^{2}\left(B_{R}\right)$. 
Proof. Recalling that $a_{\eta}=1$ in $B_{R} \backslash \mathcal{B}$, we multiply equation (1.4) by $\bar{u}_{\eta}$ (the complex conjugate of $u_{\eta}$ ) and we integrate by parts on $B_{R}$, obtaining

$$
-\int_{B_{R}} a_{\eta}(x, \tilde{\omega})\left|\nabla_{x} u_{\eta}(x, \tilde{\omega})\right|^{2} \mathrm{~d} x+k_{0}^{2} \int_{B_{R}}\left|u_{\eta}(x, \tilde{\omega})\right|^{2} \mathrm{~d} x=\int_{\partial B_{R}} u_{\eta}(x, \tilde{\omega}) \frac{\partial \overline{u_{\eta}}}{\partial n}(x, \tilde{\omega}) \mathrm{d} x .
$$

Thanks to Proposition 6.1, the right-hand side of the previous relation is uniformly bounded. Combined with (6.1) we obtain the desired upper bound. The last assertion follows from the definition of $a_{\eta}$ in (1.5).

\subsection{Derivation of the homogenized problem}

As announced in Section 2, the limit problem is characterized by two functions

$$
\mathbf{A}(x)=1_{\mathbb{R}^{2} \backslash \mathcal{B}}(x)+1_{\mathcal{B}}(x)\left(\varepsilon^{\text {eff }}\right)^{-1}, \quad \boldsymbol{b}\left(x, k_{0}\right)=1_{\mathbb{R}^{2} \backslash \mathcal{B}}(x)+\mu^{\text {eff }}\left(k_{0}\right) 1_{\mathcal{B}}(x),
$$

with $\varepsilon^{\mathrm{eff}}, \mu^{\mathrm{eff}}$ given in (2.3) and (2.8) respectively. Then we recall the homogenized problem given in (2.11)

$$
\left\{\begin{array}{l}
\operatorname{div}(\boldsymbol{A}(x) \nabla u(x))+k_{0}^{2} \boldsymbol{b}\left(x, k_{0}\right) u(x)=0, \quad x \in \mathbb{R}^{2}, \\
u-u^{\text {inc }} \quad \text { satisfies the outgoing Sommerfeld radiating condition. }
\end{array}\right.
$$

Lemma 6.3 (uniqueness). Assume that $\Im\left(\mu^{\mathrm{eff}}\right)>0$. Then the solution to $(2.11)$ is unique in $W_{\text {loc }}^{1,2}\left(\mathbb{R}^{2}\right)$.

Proof. Let $u \in W_{\text {loc }}^{1,2}$ be solving (2.11) for $u^{\text {inc }}=0$. By multiplying the first equation by $\bar{u}$ (the complex conjugate of $u$ ) and integrating by parts over $B_{R}$, we obtain:

$$
\int_{B_{R}}\left(\boldsymbol{A} \nabla u \cdot \nabla \bar{u}-k_{0}^{2} \boldsymbol{b}|u|^{2}\right) \mathrm{d} x=\int_{\partial B_{R}} \bar{u} \frac{\partial u}{\partial n} .
$$

As $u$ satisfies the outgoing wave condition (1.6), following classical arguments (see [9]), it is easy to infer that the right hand member in the equality above has a non negative imaginary part. Exploiting that $\boldsymbol{A}$ is positive definite whereas $\Im\left(\mu^{\text {eff }}\right)>0$, we deduce that $u$ vanishes a.e. on $\mathcal{B}$. Therefore by the transmission conditions in (2.13), it holds $u^{+}=\partial_{n} u^{+}=0$ on $\partial \mathcal{B}$. As $u$ solves $\left(\Delta+k_{0}^{2}\right) u=0$ on $\mathbb{R}^{2} \backslash \overline{\mathcal{B}}$, it is straightforward that $u$ vanishes over all $\mathbb{R}^{2}$.

Our second step consists in proving the following convergence result.

Proposition 6.4. Let us assume (2.10) and let $\tilde{\omega} \in \tilde{\Omega}$ such that condition (6.1) is satisfied. Then the sequence of solutions $u_{\eta}(\cdot, \tilde{\omega})$ converges weakly in $L_{\text {loc }}^{2}\left(\mathbb{R}^{2}\right)$ to $\boldsymbol{b}(\cdot) u(\cdot)$, where $u$ is the unique solution of the homogenized problem $(2.11)$.

Proof. By Lemma 6.2 and the bound (6.1), there exists $u_{0}(x, \omega) \in L^{2}\left(\mathcal{B} \times \Omega, \mathcal{L}^{2} \otimes \mathbb{P}\right)$ and vector fields $\chi_{0}(x, \omega)$, $P_{0}(x, \omega)$ in $\left(L^{2}(\mathcal{B} \times \Omega, \mathrm{d} x \otimes \mathbb{P})\right)^{2}$ such that the two-scale convergences (5.4) hold up to a subsequence. In particular for such a subsequence (still denoted with the same symbol), we have

$$
u_{\eta}(x, \tilde{\omega}) \longrightarrow u_{0}(x, \omega), \quad a_{\eta}(x, \tilde{\omega}) \nabla_{x} u_{\eta}(x, \tilde{\omega}) \longrightarrow \chi_{0}(x, \omega)
$$

where $u_{0}$ is given by Proposition 5.5 whereas $\chi_{0}(x, \cdot)$ is given by Proposition 5.8. We deduce the following weak convergences in $L^{2}\left(B_{R}\right)$ :

$$
\begin{aligned}
u_{\eta}(x, \tilde{\omega}) & \rightarrow \mathbb{E}\left(u_{0}(x, \cdot)\right)=\boldsymbol{b}(x) u(x), \\
a_{\eta}(x, \tilde{\omega}) \nabla_{x} u_{\eta}(x, \tilde{\omega}) & \rightarrow \mathbb{E}\left(\chi_{0}(x, \cdot)\right)=\mathbf{A}(x) \nabla u(x) .
\end{aligned}
$$


Then we may pass to the limit in the distributional sense in (1.4) so that $u$ solves the first equation of (2.11) in $B_{R}$. The fact that $u$ solves Helmholtz equation in $\mathbb{R}^{2} \backslash \mathcal{B}$ with $\left(u-u^{\text {inc }}\right)$ satisfying the outgoing Sommerfeld condition (1.6) is a consequence of Proposition 6.1. Summarizing we have proved that $u$ as an element of $W_{\text {loc }}^{1,2}$ solves the system (2.11). Thanks to $(2.10)$, we have $\Im\left(\mu^{\mathrm{eff}}\right)>0$ so that this limit $u$ is unique by Lemma 6.3. In particular the whole sequence $u_{\eta}(\cdot, \tilde{\omega})$ does converge weakly to $\boldsymbol{b}(\cdot) u(\cdot)$ in $L_{\text {loc }}^{2}\left(\mathbb{R}^{2}\right)$ as stated in the Proposition.

\subsection{Strong two-scale convergence and $L^{2}$-bound}

We start with a technical lemma where we use the discrete spectrum $\boldsymbol{\sigma}_{0}$ of the Dirichlet Laplace operator on the unit disk and the random positive numbers

$$
X_{j}(\omega)=\frac{1}{\operatorname{dist}\left(\varepsilon_{j}(\omega) \rho_{j}^{2}(\omega) k_{0}^{2}, \sigma_{0}\right)} .
$$

Lemma 6.5. Under condition (2.9), we have for almost every $\omega \in \Omega$ :

$$
\limsup _{\eta \rightarrow 0^{+}}\left(\eta^{2} \sum_{j \in J_{\eta}(\omega)}\left[X_{j}(\omega)\right]^{2+r}\right)<\infty, \quad \lim _{\eta \rightarrow 0^{+}}\left(\eta^{2} \sup _{j \in J_{\eta}(\omega)}\left[X_{j}(\omega)\right]^{2+r}\right)=0
$$

where the set $J_{\eta}(\omega)$ is defined in (1.3).

Proof. We notice that $\sharp\left(J_{\eta}(\omega)\right) \sim|\mathcal{B}| \eta^{-2}$. Then as the random variables $X_{j}(\omega)^{2+r}$ are independent and identically distributed, we may apply the strong law of large numbers. Thus for almost all $\omega \in \Omega$ we have

$$
\lim _{\eta \rightarrow 0} \eta^{2} \sum_{j \in J_{\eta}(\omega)}\left[X_{j}(\omega)\right]^{2+r}=\lim _{\eta \rightarrow 0} \frac{|\mathcal{B}|}{\sharp J_{\eta}(\omega)} \sum_{j \in J_{\eta}(\omega)}\left[X_{j}(\omega)\right]^{2+r}=|\mathcal{B}| \int_{M}\left[X_{j}(\omega)\right]^{2+r} d \mathbb{P}(\omega),
$$

where the right-hand side integral is finite by hypothesis (2.9). For the second part of (6.3), it is enough to apply Lemma A.5 to the sequence $\left[X_{j}(\omega)\right]^{2+r}$.

Proposition 6.6. Let us assume (2.9), (2.10) and let $\tilde{\omega} \in \tilde{\Omega}$ be such that relations (6.1) and (6.3) hold. Then for any ball $B_{R} \ni \mathcal{B}$, we have

$$
\lim _{\eta \rightarrow 0^{+}} \int_{B_{R}}\left|u_{\eta}(x, \tilde{\omega})-u_{0}\left(x, T_{\frac{x}{\eta}} \tilde{\omega}\right)\right|^{2} \mathrm{~d} x=0
$$

being $u$ the unique solution of (2.11) and $u_{0}$ given by $(2.14)$. Consequently $u_{\eta}(\cdot, \tilde{\omega})$ strongly two-scale converges to $u_{0}$ and it holds (2.15).

Proof. The proof of (6.4) with $u_{0}$ given by (2.14) follows directly from Lemmas 6.7 and 6.8 below where the convergence to zero in $L^{2}$ norm of $u_{\eta}(x, \tilde{\omega})-u_{0}\left(x, T_{\frac{x}{\eta}} \tilde{\omega}\right)$ is established on subsets $B_{R} \backslash \mathcal{D}_{\eta}$ and $\mathcal{D}_{\eta}$ respectively. The localization of (6.4) on a Borel subset $A \subset B_{R}$ and the two-scale convergence of $u_{\eta}$ to $u_{0}$ given by (2.14) leads immediately to relation (2.15).

In the rest of this Section, in order to lighten the presentation, we will use the following notations

$$
Y_{j, \eta}=\eta(j-\boldsymbol{y}(\tilde{\omega})+Y), \quad \mathcal{D}_{j, \eta}=\eta\left(j-\boldsymbol{y}(\tilde{\omega})+B\left(\boldsymbol{\theta}_{j}(\tilde{\omega}), \boldsymbol{\rho}_{j}(\tilde{\omega})\right)\right), \quad Y_{j, \eta}^{*}=Y_{j, \eta} \backslash \mathcal{D}_{j, \eta}
$$

that is we prefer to write $Y_{j, \eta}$ instead of $Y_{j, \eta}(\tilde{\omega})$ and so on for the other terms. 
Lemma 6.7. Under the assumptions of Proposition 6.6, we have

$$
\lim _{\eta \rightarrow 0^{+}}\left\|u_{\eta}(\cdot, \tilde{\omega})-u\right\|_{L^{p}\left(B_{R} \backslash \mathcal{D}_{\eta}\right)}=0, \quad \forall p \in[1, \infty) .
$$

Proof. We modify $u_{\eta}$ in the subset $\mathcal{D}_{\eta}(\tilde{\omega})$ by considering the harmonic extension. This way we obtain the new function $\tilde{u}_{\eta} \in H^{1}\left(B_{R}\right)$ defined by

$$
\begin{cases}\tilde{u}_{\eta}(x)=u_{\eta}(x, \tilde{\omega}), & x \in B_{R} \backslash \mathcal{D}_{\eta} \\ \Delta \tilde{u}_{\eta}(x)=0 & x \in \mathcal{D}_{\eta},\end{cases}
$$

By the rescaled inequality (A.5), we have

$$
\begin{aligned}
\left\|\tilde{u}_{\eta}\right\|_{H^{1}\left(B_{R}\right)}^{2} & =\left\|u_{\eta}(\cdot, \tilde{\omega})\right\|_{H^{1}\left(B_{R} \backslash \mathcal{D}_{\eta}\right)}^{2}+\sum_{i \in J_{\eta}}\left\|\tilde{u}_{\eta}\right\|_{H^{1}\left(\mathcal{D}_{j, \eta}\right)}^{2} \\
& \leq\left\|u_{\eta}(\cdot, \tilde{\omega})\right\|_{H^{1}\left(B_{R} \backslash \mathcal{D}_{\eta}\right)}^{2}+c_{\delta} \sum_{i \in J_{\eta}}\left\|\tilde{u}_{\eta}\right\|_{H^{1}\left(Y_{j, \eta}^{*}\right)}^{2}=\left(1+c_{\delta}\right)\left\|u_{\eta}(\cdot, \tilde{\omega})\right\|_{H^{1}\left(B_{R} \backslash \mathcal{D}_{\eta}\right)}^{2} \\
& \leq\left(1+c_{\delta}\right) \int_{B_{R}}\left(\left|u_{\eta}(x, \tilde{\omega})\right|^{2}+a_{\eta}(x, \tilde{\omega})\left|\nabla u_{\eta}(x, \tilde{\omega})\right|^{2}\right) \mathrm{d} x .
\end{aligned}
$$

By (6.1) and the estimate in Lemma 6.2, the last integral is uniformly bounded. Therefore, by the Sobolev compact embedding $H^{1}\left(B_{R}\right) \subset L^{p}\left(B_{R}\right)$ for $p \in[1, \infty)$, we can extract a convergent sequence (still denoted by $\left.\tilde{u}_{\eta}\right)$ such that $\tilde{u}_{\eta} \rightarrow \tilde{u}$ in $L^{p}\left(B_{R}\right)$. It remains to check that $\tilde{u}=u$ in $\mathcal{B}$. To that aim we observe that $\tilde{u}_{\eta}-u_{\eta} \longrightarrow \tilde{u}(x)-u_{0}(x, \omega)$ whereas we have the strong two-scale convergence $1_{\mathcal{B} \backslash \mathcal{D}_{\eta}(\tilde{\omega})}(x) \rightarrow 1_{\mathcal{B} \times \Sigma^{*}}(x, y)$. Therefore by applying the product rule (5.3), we deduce the following convergence in distributional sense

$$
\left(\tilde{u}_{\eta}-u_{\eta}\right) 1_{\mathcal{B} \backslash \mathcal{D}_{\eta}(\tilde{\omega})} \rightarrow \mathbb{E}\left(\left(\tilde{u}(x)-u_{0}(x, \omega)\right) 1_{\mathcal{B} \times \Sigma^{*}}\right)=\mathbb{E}\left(\Sigma^{*}\right)(\tilde{u}(x)-u(x))
$$

where the last equality comes from $u_{0}(x, \omega)=u(x)$ in $\Sigma^{*}$. As equality $\tilde{u}_{\eta}(x)=u_{\eta}(x, \tilde{\omega})$ hold in $\mathcal{B} \backslash \mathcal{D}_{\eta}$, we conclude that $\tilde{u}=u$ a.e. in $\mathcal{B}$.

The proof of next Lemma is particularly involved. We will need a general estimate established in the Appendix (Lem. A.3) that we are going to use in the rescaled form (A.6).

Lemma 6.8. Under the assumptions of Proposition 6.6, it holds

$$
\lim _{\eta \rightarrow 0^{+}}\left\|u_{\eta}(x, \tilde{\omega})-u_{0}\left(x, T_{\frac{x}{\eta}} \tilde{\omega}\right)\right\|_{L^{2}\left(\mathcal{D}_{\eta}\right)}=0 .
$$

Proof. Let us set $v_{\eta}(x)=u_{\eta}(x, \tilde{\omega})-u_{0}\left(x, T_{\frac{x}{\eta}} \tilde{\omega}\right)$. We write $\left\|v_{\eta}\right\|_{L^{2}\left(\mathcal{D}_{\eta}\right)}^{2}=\sum_{j \in J_{\eta}}\left\|v_{\eta}\right\|_{L^{2}\left(\mathcal{D}_{j, \eta}\right)}^{2}$ and, for every $j \in J_{\eta}$, we apply to $v_{\eta}$ the rescaled inequality (A.6) on each disk $\mathcal{D}_{j, \eta}$ choosing $\alpha=\varepsilon_{j} k_{0}^{2}$. We get

$$
\begin{aligned}
\left\|v_{\eta}\right\|_{L^{2}\left(\mathcal{D}_{\eta}\right)}^{2} \leq & \sum_{j} \frac{2 \rho_{j}^{2} \eta^{4}}{\operatorname{dist}^{2}\left(\varepsilon_{j} \rho_{j}^{2} k_{0}^{2}, \boldsymbol{\sigma}_{0}\right)}\left\|\Delta v_{\eta}+\frac{\varepsilon_{j} k_{0}^{2}}{\eta^{2}} v_{\eta}\right\|_{L^{2}\left(\mathcal{D}_{j, \eta}\right)}^{2} \\
& +4 c_{\delta} \sum_{j}\left(\frac{\left|\varepsilon_{j}\right|^{2} k_{0}^{4} \rho_{j}^{4}}{\operatorname{dist}^{2}\left(\varepsilon_{j} k_{0}^{2} \rho_{j}^{2}, \boldsymbol{\sigma}_{0}\right)}+1\right)\left(\left\|v_{\eta}\right\|_{L^{2}\left(Y_{j, \eta}^{*}\right)}^{2}+\eta^{2}\left\|\nabla v_{\eta}\right\|_{L^{2}\left(Y_{j, \eta}^{*}\right)}^{2}\right) .
\end{aligned}
$$

As $\tilde{\omega}$ satisfies (6.3), we have the following convergence

$$
b_{\eta}:=\sup _{j \in J_{\eta}} \eta^{2}\left[X_{j}(\tilde{\omega})\right]^{2} \rightarrow 0
$$


On the other hand, recalling that the $\left(\rho_{j}, \varepsilon_{j}\right)$ remain in a bounded set of $\mathbb{R}^{+} \times \mathbb{C}$ and that $\varepsilon_{j}=\varepsilon_{0}\left(T_{\frac{x}{\eta}} \tilde{\omega}\right)$ for $x \in \mathcal{D}_{j, \eta}$, we deduce from (6.6) the existence of a suitable constant $\kappa>0$ (depending only on $\delta$ ) such that

$$
\begin{aligned}
\kappa\left\|v_{\eta}\right\|_{L^{2}\left(\mathcal{D}_{\eta}\right)}^{2} \leq\left(b_{\eta}+\eta^{2}\right)\left\|\nabla v_{\eta}\right\|_{L^{2}\left(B_{R} \backslash \mathcal{D}_{\eta}\right)}^{2}+\left\|v_{\eta}\right\|_{L^{2}\left(B_{R} \backslash \mathcal{D}_{\eta}\right)}^{2} & \\
& \quad+\sum_{i \in J_{\eta}}\left(X_{j}^{2}(\omega) \int_{Y_{j, \eta}^{*}}\left|v_{\eta}(x)\right|^{2}\right)+b_{\eta} \eta^{2}\left\|\Delta v_{\eta}+\frac{\varepsilon_{0}(T \cdot / \eta \tilde{\omega}) k_{0}^{2}}{\eta^{2}} v_{\eta}\right\|_{L^{2}\left(\mathcal{D}_{\eta}\right)}^{2} .
\end{aligned}
$$

Therefore, in view of (6.7) and (6.8), the Lemma is proved provided one shows the following claims

$$
\begin{array}{ll}
\text { i) } & \left\|v_{\eta}\right\|_{L^{2}\left(B_{R} \backslash \mathcal{D}_{\eta}\right)}^{2} \rightarrow 0 ; \\
\text { ii) } & \sup _{\eta>0}\left\|\nabla v_{\eta}\right\|_{L^{2}\left(B_{R} \backslash \mathcal{D}_{\eta}\right)}^{2}<\infty ; \\
\text { iii) } & \sup _{\eta>0} \eta^{2}\left\|\Delta v_{\eta}+\frac{\varepsilon_{0}\left(T_{\overline{\bar{\eta}}} \tilde{\omega}\right) k_{0}^{2}}{\eta^{2}} v_{\eta}\right\|_{L^{2}\left(\mathcal{D}_{\eta}\right)}^{2}<+\infty ; \\
\text { iv) } & \sum_{j \in J_{\eta}}\left(X_{j}^{2}(\omega) \int_{Y_{j, \eta}^{*}}\left|v_{\eta}(x)\right|^{2}\right) \rightarrow 0 .
\end{array}
$$

Recalling that $T_{\frac{x}{\eta}} \tilde{\omega} \in \Sigma^{*}$ for $x \in B_{R} \backslash \mathcal{D}_{\eta}$ (see (4.3)) while $u_{0}\left(x, T_{\frac{x}{\eta}} \tilde{\omega}\right)=u(x)$ for $x \in B_{R} \backslash \mathcal{D}_{\eta}$ (see Prop. 5.5, i)), claim i) follows directly from Lemma 6.7. On the other hand as $\nabla v_{\eta}(x)=\nabla u_{\eta}(x, \tilde{\omega})-\nabla u(x)$ holds in $B_{R} \backslash \mathcal{D}_{\eta}$, we deduce claim ii) taking into account that $u \in H^{1}\left(B_{R}\right)$ (see Prop. 5.8) and the gradient estimates in Lemma 6.2.

Let us prove now claim iii). As $u_{\eta}$ solves $(2.3)$, we get for $x \in \mathcal{D}_{j, \eta}(\tilde{\omega})$ :

$$
\begin{aligned}
\Delta v_{\eta}(x)+\frac{\varepsilon_{j} k_{0}^{2}}{\eta^{2}} v_{\eta}(x) & =\Delta_{x} u_{\eta}+a_{\eta}(x, \tilde{\omega}) u_{\eta}-\Delta_{x}\left(u_{0}\left(x, T_{\frac{x}{\eta}} \tilde{\omega}\right)\right)-\frac{\varepsilon_{j} k_{0}^{2}}{\eta^{2}} u_{0}\left(x, T_{\frac{x}{\eta}} \tilde{\omega}\right) \\
& =-\Delta_{x}\left(u_{0}\left(x, T_{\frac{x}{\eta}} \tilde{\omega}\right)\right)-\frac{\varepsilon_{j} k_{0}^{2}}{\eta^{2}} u_{0}\left(x, T_{\frac{x}{\eta}} \tilde{\omega}\right) .
\end{aligned}
$$

On the other hand, thanks to relation $u_{0}(\cdot, \omega)=u(\cdot) \Lambda(\omega)$ in $\mathcal{B}$ and the chain rule (4.11) it comes

$$
\Delta_{x}\left(u_{0}\left(x, T_{\frac{x}{\eta}} \tilde{\omega}\right)\right)=\Delta_{x} u(x) \Lambda\left(T_{\frac{x}{\eta}} \tilde{\omega}\right)+\frac{2}{\eta} \nabla u(x) \cdot \nabla^{s} \Lambda\left(T_{\frac{x}{\eta}} \tilde{\omega}\right)+\frac{1}{\eta^{2}} u(x) \Delta_{s} \Lambda\left(T_{\frac{x}{\eta}} \tilde{\omega}\right) .
$$

Plugging (6.9) and (6.10) yields

$$
\begin{aligned}
\Delta v_{\eta}(x)+\frac{\varepsilon_{j} k_{0}^{2}}{\eta^{2}} v_{\eta}(x)= & -\Delta_{x} u(x) \Lambda\left(T_{\frac{x}{\eta}} \tilde{\omega}\right)-\frac{2}{\eta} \nabla u(x) \cdot \nabla^{s} \Lambda\left(T_{\frac{x}{\eta}} \tilde{\omega}\right) \\
& -\frac{1}{\eta^{2}}\left(\Delta_{s} u_{0}\left(x, T_{\frac{x}{\eta}} \tilde{\omega}\right)-\varepsilon_{j} k_{0}^{2} u_{0}\left(x, T_{\frac{x}{\eta}} \tilde{\omega}\right)\right) .
\end{aligned}
$$

Recalling that $\left(T_{x / \eta} \tilde{\omega}\right) \in \Sigma$ (see (4.3)) and that $u_{0}$ solves the equation appearing in assertion ii) of Proposition 5.5, we deduce after multipling by $\eta$

$$
\eta\left(\Delta v_{\eta}(x)+\frac{\varepsilon_{j} k_{0}^{2}}{\eta^{2}} v_{\eta}(x)\right)=-\eta \Delta_{x} u(x) \Lambda\left(T_{\frac{x}{\eta}} \tilde{\omega}\right)-2 \nabla u(x) \cdot \nabla^{s} \Lambda\left(T_{\frac{x}{\eta}} \tilde{\omega}\right) .
$$

By Lemma 5.7, we have $\Lambda \in H_{s}^{1}(\Omega)$ from which follows that functions $x \mapsto \Delta_{x} u(x) \Lambda\left(T_{\frac{x}{\eta}} \tilde{\omega}\right)$ and $x \mapsto$ $\nabla u(x) \cdot \nabla^{s} \Lambda\left(T_{\frac{x}{\eta}} \tilde{\omega}\right)$ are bounded in $L^{2}(\Omega)$ (and strongly two-scale converge to $\Delta_{x} u(x) \Lambda(\omega)$ and $u(x) \cdot \nabla^{s} \Lambda(\omega)$ respectively). Thus claim iii) is a consequence of (6.11). 
It remains to show claim iv). Let $q=(2+r) / 2$, being $r$ the parameter introduced in the hypothesis (2.9) and let $q^{*}$ be such that $q^{-1}+q^{*-1}=1$. By applying Hölder inequality first on the sum over $j \in J_{\eta}$ and then on the integral, it comes

$$
\begin{aligned}
\sum_{j \in J_{\eta}} X_{j}(\omega)^{2} \int_{Y_{j, \eta}^{*}(\omega)}\left|v_{\eta}\right|^{2} & \leq\left[\sum_{j \in J_{\eta}} X_{j}(\omega)^{2 q}\right]^{\frac{1}{q}}\left[\sum_{j \in J_{\eta}}\left(\int_{Y_{j, \eta}^{*}}\left|v_{\eta}\right|^{2}\right)^{q^{*}}\right]^{\frac{1}{q^{*}}} \\
& \leq\left(\sum_{j \in J_{\eta}(\omega)} \eta^{2} X_{j}(\omega)^{2 q}\right)^{\frac{1}{q}}\left\|v_{\eta}\right\|_{L^{2 q^{*}}\left(B_{R} \backslash \mathcal{D}_{\eta}\right)}^{2} .
\end{aligned}
$$

By (6.3) the first factor on the right hand side remains bounded as $\eta \rightarrow 0^{+}$while the second factor vanishes in virtue of Lemma 6.7. This implies claim iv). The proof of Lemma 6.8 is complete.

The last issue before completing the third and last step of the proof of Theorem 2.3 consists in proving the energy bound (6.1). As announced it will be done by using a contradiction argument.

Proposition 6.9 (energy bound). Assume that (2.9) and (2.10) hold. Then the bound (6.1) holds $\mathbb{P}$-almost surely.

Proof. Let $\mathcal{Q} \subset \Omega$ be the set of all $\omega$ such that (6.3) holds. As by Lemma 6.5, $\mathcal{Q}$ is of full measure, it is enough to show that the bound (6.1) holds true for every $\omega \in \mathcal{Q}$. Assume by contradiction that (6.1) does not hold for some particular $\tilde{\omega} \in \mathcal{Q}$. Then there exists a sequence $\eta_{j} \searrow 0, j \in \mathbb{N}$ such that

$$
t_{j}=\left\|u_{\eta_{j}}(\cdot, \tilde{\omega})\right\|_{L^{2}\left(B_{R}\right)} \nearrow \infty \quad \text { as } j \rightarrow \infty .
$$

By the linearity of the problem, the normalized function $v_{j}=\frac{u_{\eta_{j}}(\cdot, \tilde{\omega})}{t_{j}}$ satisfies

$$
\left\{\begin{array}{l}
\operatorname{div}\left(a_{\eta_{j}}(x, \tilde{\omega}) \nabla v_{j}(x)\right)+k_{0}^{2} v_{j}(x)=0 \quad x \in \mathbb{R}^{2} \\
\left\|v_{j}\right\|_{L^{2}\left(B_{R}\right)}=1, \\
v_{j}-\frac{u^{\text {inc }}}{t_{j}} \text { satisfies the Sommerfeld radiating condition. }
\end{array}\right.
$$

This means that $v_{j}$ solves the diffraction problem (1.4) for $\eta=\eta_{j}$ and a incident wave $\frac{u^{\text {inc }}}{t_{j}}$ tending to zero as $j \rightarrow \infty$. As by construction $\left\{v_{j} ; j \in \mathbb{N}\right\}$ is bounded in $L^{2}\left(B_{R}\right)$, we may apply Proposition 6.6 and we are led to

$$
\lim _{j \rightarrow \infty} \int_{B_{R}}\left|v_{j}(x, \tilde{\omega})-v_{0}\left(x, T_{x / \eta} \tilde{\omega}\right)\right|^{2} \mathrm{~d} x=0,
$$

where $v_{0}(x, \omega)=v(x)\left[1_{\mathbb{R}^{2} \backslash \mathcal{B}}(x)+\Lambda(\omega) 1_{\mathcal{B}}(x)\right]$ being $v$ the solution of $(2.11)$ for a vanishing incident wave. Here the random function $\Lambda$ is the one defined in (2.7). By the uniqueness Lemma 6.3 , we have $v \equiv 0$, thus $v_{0} \equiv 0$ which is incompatible with (6.12) and equality $\left\|v_{j}\right\|=1$.

\section{Appendix A.}

In the following Lemma we keep the notations of Section 2 for the spectral Dirichlet problem in the unit disk $D$ of $\mathbb{R}^{2}$. We denote $B_{\rho}=\rho D=B(0, \rho)$.

Lemma A.1 (Resonator problem). Let $\rho>0$ and $\alpha \in \mathbb{C}$ such that $\rho^{2} \alpha \notin \boldsymbol{\sigma}_{0}$. Then

i) The boundary value problem $\Delta w+\alpha w=0, w=1$ on $\partial B_{\rho}$ has a unique solution $w \in W^{1,2}\left(B_{\rho}\right)$ given by

$$
w(y)=1+\sum_{n=1}^{+\infty} \frac{\alpha \rho^{2} c_{n}}{\lambda_{n}-\alpha \rho^{2}} \varphi_{n}\left(\frac{y}{\rho}\right), \quad \text { with } c_{n}=\int_{D} \varphi_{n} .
$$


ii) For all $v \in W_{0}^{1,2}\left(B_{\rho}\right) \cap W^{2,2}\left(B_{\rho}\right)$, it holds

$$
\|\Delta v+\alpha v\|_{L^{2}\left(B_{\rho}\right)} \geq \operatorname{dist}\left(\alpha, \frac{\boldsymbol{\sigma}_{0}}{\rho^{2}}\right)\|v\|_{L^{2}\left(B_{\rho}\right)} .
$$

Proof. Straightforward by using the spectral decomposition of the Laplace operator on $B_{\rho}$ whose eigenvalues are $\left\{\frac{\lambda_{n}}{\rho^{2}}\right\}$ with normalized eigenvectors $\left\{\frac{1}{\sqrt{\rho}} \varphi_{n}\left(\frac{x}{\rho}\right)\right\}$.

Lemma A.2. Let $\delta \in(0,1 / 2)$. Then there exists $c_{\delta}>0$ such that, for every $(\theta, \rho)$ with $\operatorname{dist}(\theta, \partial Y) \geq \rho+\delta$ and for every $u \in W^{1,2}(Y)$ verifying $\Delta u=0$ in $B(\theta, \rho)$, there holds

$$
\begin{gathered}
\int_{B(\theta, \rho)}|\nabla u|^{2} \leq c_{\delta} \int_{Y \backslash B(\theta, \rho)}|\nabla u|^{2} . \\
\int_{B(\theta, \rho)}|u|^{2} \leq c_{\delta} \int_{Y \backslash B(\theta, \rho)}\left(|u|^{2}+|\nabla u|^{2}\right) .
\end{gathered}
$$

Proof. In a first step we show the result assuming that $B(\theta, \rho)$ is the largest ball in $Y$ such that dist $(\theta, \partial Y) \geq \rho+\delta$ namely $\bar{B}=B\left(\bar{\theta}, \frac{1}{2}-\delta\right)$ with $\bar{\theta}=\left(\frac{1}{2}, \frac{1}{2}\right)$.

Proof of (A.2): Assume by contradiction that (A.2) does not hold. Then there exists a sequence $\left(u_{n}\right) \subset W^{1,2}(Y)$ such that

$$
\int_{\bar{B}}\left|\nabla u_{n}\right|^{2}=1, \quad \int_{Y \backslash \bar{B}}\left|\nabla u_{n}\right|^{2} \rightarrow 0, \quad \Delta u_{n}=0 \text { in } \bar{B} .
$$

Without any loss of generality we may assume additionally that $\int_{Y} u_{n}=0$. Thus, by Poincaré inequality, $\left\{u_{n}\right\}$ is bounded in $W^{1,2}(Y)$ and, possibly after extracting a subsequence, does converge strongly in $L^{2}(Y)$ to some limit $u \in W^{1,2}(Y)$. From (A.4) it comes that $\nabla u=0$ in $Y \backslash \bar{B}$ while $\Delta u=0$ in $B$. Therefore $u$ is constant and vanishes by the zero average condition. In addition the convergence $u_{n}$ to 0 over $Y \backslash \bar{B}$ is strong in $W^{1,2}(Y \backslash \bar{B})$ yielding in particular that the trace of $u_{n}$ does converge strongly to zero in $W^{1 / 2,2}(\partial B)$. As $u_{n}$ is harmonic in $\bar{B}$, we deduce that $\int_{\bar{B}}\left|\nabla u_{n}\right|^{2} \rightarrow 0$ in contradiction with (A.4).

Proof of (A.3): Assume by contradiction that (A.3) does not hold. Then there exists a sequence $\left(u_{n}\right) \subset W^{1,2}(Y)$ such that

$$
\int_{\bar{B}}\left|u_{n}\right|^{2}=1, \quad \int_{Y \backslash \bar{B}}\left|u_{n}\right|^{2}+\left|\nabla u_{n}\right|^{2} \rightarrow 0, \quad \Delta u_{n}=0 \text { in } \bar{B} .
$$

By (A.2), we deduce that $\left(u_{n}\right)$ is bounded in $W^{1,2}(Y)$ and satisfies $\nabla u_{n} \rightarrow 0$ in $L^{2}(Y)$ while $u_{n} \rightarrow 0$ in $L^{2}(Y \backslash \bar{B})$. It follows that $u_{n} \rightarrow 0$ strongly in $W^{1,2}(Y)$ in contradiction with $\int_{\bar{B}}\left|u_{n}\right|^{2}=1$.

So far we have proved (A.2) and (A.3) if $B(\theta, \rho)=B(\bar{\theta}, 1 / 2-\delta)$. Let us consider the general case of $\theta \in Y$ and $\rho<1 / 2$ such that $\operatorname{dist}(\theta, \partial Y) \geq \rho+\delta$. It is easy to see that

$$
B(\theta, \rho)=\theta+t\left(\bar{B}-\left(\frac{1}{2}, \frac{1}{2}\right)\right) \quad \text { with } \quad t=\frac{\rho}{\frac{1}{2}-\delta} .
$$

With the same transformation, we define $Y_{\theta, \rho}:=\theta+t\left(Y-\left(\frac{1}{2}, \frac{1}{2}\right)\right)$. Clearly, by affine change of variable, inequalities (A.2) and (A.3) still hold true when we substitute $Y$ with $Y_{\theta, \rho}$ and $\bar{B}$ with $B(\theta, \rho)$. For instance, the same constant $c_{\delta}$ appears provided the homothety factor $t$ satisfies $t \leq 1$. The general case follows by observing that we have the inclusion $Y_{\theta, \rho} \backslash B(\theta, \rho) \subset Y \backslash B(\theta, \rho)$.

Lemma A.3. Let $c_{\delta}>0$ given in Lemma A.2 and $(\theta, \rho)$ with $\operatorname{dist}(\theta, \partial Y) \geq \rho+\delta$. Then for every $u \in W^{1,2}(Y)$ verifying $\Delta u \in L^{2}(B(\theta, \rho))$, there holds

$$
\int_{B}|u|^{2} \leq \frac{2}{\operatorname{dist}^{2}\left(\alpha, \frac{\sigma_{0}}{\rho^{2}}\right)} \int_{B(\theta, \rho)}|\Delta u+\alpha u|^{2}+4 c_{\delta}\left(1+\frac{|\alpha|^{2}}{\operatorname{dist}^{2}\left(\alpha, \frac{\sigma_{0}}{\rho^{2}}\right)}\right) \int_{Y \backslash B(\theta, \rho)}\left(|u|^{2}+|\nabla u|^{2}\right) .
$$


Proof. We set $B=B(\theta, \rho)$ and use for every $u \in W^{1,2}(Y)$ the decomposition $u=u_{1}+u_{2}$, where

$$
\Delta u_{1}=0 \quad \text { on } B, \quad u_{1}=u \quad \text { on } Y \backslash B, \quad u_{2}:=u-u_{1} .
$$

Then $u_{2} \in W_{0}^{1,2}(B)$ and as $\Delta u_{2}=\Delta u$ in the distribution sense on open subset $B$, we have that $u_{2} \in W^{2,2}(B)$ and by applying Lemma A.1

$$
\operatorname{dist}\left(\alpha, \rho^{-2} \boldsymbol{\sigma}_{0}\right)\left\|u_{2}\right\|_{L^{2}(B)} \leq\left\|\Delta u+\alpha\left(u-u_{1}\right)\right\|_{L^{2}(B)} \leq\|\Delta u+\alpha u\|_{L^{2}(B)}+|\alpha|\left\|u_{1}\right\|_{L^{2}(B)} .
$$

Then by triangle inequality

$$
\|u\|_{L^{2}(B)} \leq \frac{1}{\operatorname{dist}\left(\alpha, \rho^{-2} \boldsymbol{\sigma}_{0}\right)}\|\Delta u+\alpha u\|_{L^{2}(B)}+\left(1+\frac{|\alpha|}{\operatorname{dist}\left(\alpha, \rho^{-2} \boldsymbol{\sigma}_{0}\right)}\right)\left\|u_{1}\right\|_{L^{2}(B)} .
$$

The conclusion follows by applying Lemma A.2 to $u_{1}$ noticing that $u_{1}=u$ on $Y \backslash B$.

Remark A.4. In Lemmas A.2 and A.3 the constant $c_{\delta}$ blows up to infinity when $\delta \rightarrow 0$. We will apply both lemmas in the infinitesimal domain $\eta Y$ in the form of the following rescaled inequalities. Let $\eta>0$ and let $\varphi \in W^{1,2}(\eta Y)$ such that $\Delta \varphi \in L^{2}(\eta B(\theta, \rho))$. Then, provided dist $(\theta, \partial Y) \geq \rho+\delta$, one has

$$
\begin{gathered}
\int_{\eta B(\theta, \rho)}|\varphi|^{2} \leq c_{\delta} \int_{\eta(Y \backslash B(\theta, \rho))}|\varphi|^{2}+\eta^{2}|\nabla \varphi|^{2}, \quad \int_{\eta B(\theta, \rho)}|\varphi|^{2} \leq c_{\delta} \int_{\eta(Y \backslash B(\theta, \rho))}|\nabla \varphi|^{2}, \\
\int_{\eta B(\theta, \rho)}|\varphi|^{2} \leq 4 c_{\delta}\left(1+\frac{|\alpha|^{2}}{\operatorname{dist}^{2}\left(\alpha, \frac{\sigma_{0}}{\rho^{2}}\right)}\right) \int_{\eta(Y \backslash B(\theta, \rho))}\left(|\varphi|^{2}+\eta^{2}|\nabla \varphi|^{2}\right) \\
+\frac{2 \eta^{4}}{\operatorname{dist}^{2}\left(\alpha, \frac{\sigma_{0}}{\rho^{2}}\right)} \int_{\eta B(\theta, \rho)}\left|\Delta \varphi+\frac{\alpha}{\eta^{2}} \varphi\right|^{2} .
\end{gathered}
$$

Lemma A.5. Let $\left\{X_{i}, i=1,2, \ldots, n, \ldots\right\}$ be a sequence of identically distributed non negative random variables in $L^{1}(\Omega, \mathcal{A}, \mathbb{P})$. Define $Z_{n}:=\sup \left\{X_{i}, 1 \leq i \leq n\right\}$. Then it holds: $\frac{Z_{n}}{n} \stackrel{\text { a.s. }}{\longrightarrow} 0$ and $\lim _{n \rightarrow \infty} \frac{1}{n} \mathbb{E}\left(Z_{n}\right)=0$.

Proof. Let $\varepsilon>0$. Noticing that the function $t \mapsto \mathbb{P}\left(\left\{X_{n} \geq t \varepsilon\right\}\right)$ is non increasing on $\mathbb{R}^{+}$and does not depend on $n$, we have:

$$
\sum_{1}^{\infty} \mathbb{P}\left(\left\{\frac{X_{n}}{n}>\varepsilon\right\}\right)=\sum_{1}^{\infty} \mathbb{P}\left(\left\{\frac{X_{1}}{n}>\varepsilon\right\}\right) \leq \int_{0}^{+\infty} \mathbb{P}\left(\left\{X_{1}>t \varepsilon\right\}\right) \mathrm{d} t=\frac{1}{\varepsilon} \mathbb{E}\left(X_{1}\right)<+\infty .
$$

By Borel-Cantelli lemma, it follows that $\mathbb{P}\left(\lim \sup _{n}\left\{\frac{X_{n}}{n}>\varepsilon\right\}\right)=0$. Thus for a.a. $\omega$ there exists a finite integer $N(\omega)$ such that:

$$
\frac{Z_{n}(\omega)}{n} \leq \max \left\{\frac{Z_{N}(\omega)}{n}, \varepsilon\right\}, \forall n \geq N(\omega)
$$

We may assume that $Z_{N}(\omega)$ is finite. By letting $n \rightarrow \infty$ and then $\varepsilon \rightarrow 0$, we conclude that $\frac{Z_{n}}{n} \stackrel{\text { a.s. }}{\longrightarrow} 0$. Furthermore, since $\left\{Z_{n}>t\right\} \subset \bigcup_{i=1}^{n}\left\{X_{i}>t\right\}$ hold for every $t$, the function $f_{n}(t):=\frac{\mathbb{P}\left(\left\{Z_{n}>t\right\}\right)}{n}$ satisfies the upperbound $f_{n}(t) \leq f(t):=\mathbb{P}\left(\left\{X_{1}>t\right\}\right)$. As $\int_{0}^{\infty} f(t)=\mathbb{E}\left(X_{1}\right)<+\infty$, we infer by dominated convergence that $\lim _{n \rightarrow \infty} \frac{\mathbb{E}\left(Z_{n}\right)}{n}=\lim _{n \rightarrow \infty} \int_{0}^{\infty} f_{n}(t) \mathrm{d} t=0$.

Acknowledgements. This work was initiated while the third author was supported by ANR grant POEM ANR-06-NANO008-02. The final steps of the paper have been completed while the first two authors were supported by ANR grants OPTRANS-2010-BLAN-0124 and METAMATH-11 MONU-01604. 


\section{REFERENCES}

[1] G. Allaire, Homogenization and two-scale convergence. SIAM J. Math. Anal. 23 (1992) 1482-1518.

[2] G. Bouchitté and C. Bourel, Multiscale nanorod metamaterials and realizable permittivity tensors. Commun. Comput. Phys. 11 (2012) 489-507.

[3] G. Bouchitté and D. Felbacq, Homogenization near resonances and artificial magnetism from dielectrics. C. R. Math. Acad. Sci. Paris 339 (2004) 377-382.

[4] G. Bouchitté and D. Felbacq, Homogenization of a wire photonic crystal: the case of small volume fraction. SIAM J. Appl. Math. 66 (2006) 2061-2084.

[5] G. Bouchitté and B. Schweizer, Homogenization of Maxwell's equations in a split ring geometry. Multiscale Model. Simul. 8 (2010) 717-750.

[6] G. Bouchitté, C. Bourel and D. Felbacq, Homogenization of the 3D Maxwell system near resonances and artificial magnetism. C. R. Math. Acad. Sci. Paris 347 (2009) 571-576.

[7] A. Bourgeat, A. Mikelić and S. Wright, Stochastic two-scale convergence in the mean and applications. J. Reine Angew. Math. 456 (1994) 19-51.

[8] D. Cioranescu and J.S.J. Paulin, Homogenization in open sets with holes. J. Math. Anal. Appl. 71 (1979) 590-607.

[9] D. Colton and R. Kress, Inverse acoustic and electromagnetic scattering theory. Vol. 93 of Appl. Math. Sci. Springer-Verlag, Berlin, 2nd edition (1998).

[10] D.J. Daley and D. Vere-Jones, An introduction to the theory of point processes. General theory and structure. Vol. 2 of Probab. Appl. Springer, New York, 2nd edition (2008).

[11] D. Felbacq and G. Bouchitté, Theory of mesoscopic magnetism in photonic crystals. Phys. Rev. Lett. 94 (2005) 183902.

[12] R.M. Dudley. Real analysis and probability, Mathematics Series. Wadsworth \& Brooks/Cole Advanced Books \& Software, Pacific Grove, CA (1989).

[13] D. Felbacq, G. Tayeb and D. Maystre, Scattering by a random set of parallel cylinders. J. Optim. Soc. Am. A 11 (1994) $2526-2538$.

[14] D. Felbacq and G. Bouchitté, Negative refraction in periodic and random photonic crystals. New J. Phys. 7 (2005) 159.

[15] G. Bouchitté and D. Felbacq, Homogenization of a set of parallel fibers. Waves in Random Media 7 (1997) 1-12.

[16] A.A. Houck, J.B. Brock and I.L. Chuang, Experimental observations of a left-handed material that obeys snell's law. Phys. Rev. Lett. 90137401 (2003).

[17] V.V. Jikov, S.M. Kozlov and O.A. Oleŭnik, Homogenization of differential operators and integral functionals. Springer-Verlag, Berlin (1994).

[18] R.V. Kohn and S.P. Shipman, Magnetism and homogenization of microresonators. Multiscale Model. Simul. 7 (2008) 62-92.

[19] G. Nguetseng, A general convergence result for a functional related to the theory of homogenization. SIAM J. Math. Anal. 20 (1989) 608-623.

[20] S. O'Brien and J.B. Pendry, Magnetic activity at infrared frequencies in structured metallic photonic crystals. J. Phys.: Condensed Matter 14 (2002) 6383-6394.

[21] S. O'Brien and J.B. Pendry, Photonic band-gaps effects and magnetic activity in dielectric composites. J. Phys.: Condensed Matter 14 (2002) 4035.

[22] J.B. Pendry, A.J. Holden, D.J. Robbins and W.J. Stewart, Magnetism from conductors and enhanced nonlinear phenomena. Microwave Theory and Techniques. IEEE Trans. 47 (1999) 2075-2084.

[23] R.A. Shelby, D.R. Smith and S. Schultz, Experimental verification of a negative index of refraction. Science 292 (2001) 77-79.

[24] D.R. Smith, Willie J. Padilla, D.C. Vier, S.C. Nemat-Nasser and S. Schultz, Composite medium with simultaneously negative permeability and permittivity. Phys. Rev. Lett. 84 (2000) 4184-4187.

[25] V.G. Veselago, The electrodynamics of substances with simultaneously negative values of $\epsilon$ and $\mu$. Soviet Phys. Usp. 10 (1968) 509.

[26] V.V. Zhikov and A.L. Piatnitski, Homogenization of random singular structures and random measures. Izv. Ross. Akad. Nauk Ser. Mat. 70 (2006) 23-74. 